Article

\title{
MADM strategy based on some similarity measures in interval bipolar neutrosophic set environment
}

\author{
Surapati Pramanik ${ }^{1,}{ }^{*}$, Partha Pratim Dey ${ }^{2}$ and Florentin Smarandache ${ }^{3}$ \\ 1 Department of Mathematics, Nandalal Ghosh B.T. College, Panpur, P.O.-Narayanpur, District -North 24 Parganas, Pin \\ code-743126,West Bengal, India; sura_pati@yahoo.co.in \\ 2 Patipukur Pallisree Vidyapith, Department of Mathematics, Patipukur, Kolkata-700048, West Bengal, India; \\ parsur.fuzz@gmail.com \\ 3 University of New Mexico, Mathematics \& Science Department, 705Gurley Ave., Gallup, NM 87301, U.S.A.; \\ smarand@unm.edu \\ * Correspondence: sura_pati@yahoo.co.in; Tel.: +91-947-703-5544
}

\begin{abstract}
The paper investigates some similarity measures in interval bipolar neutrosophic environment for multi-attribute decision making problems. At first, we define Hamming and Euclidean distances measures between interval bipolar neutrosophic sets and establish their basic properties. We also propose two similarity measures based on the Hamming and Euclidean distance functions. Using maximum and minimum operators, we define new similarity measures and prove their basic properties. Using the proposed similarity measures, we propose a novel multi attribute decision making strategy in interval bipolar neutrosophic set environment. Lastly, we solve an illustrative example of multi attribute decision making and present comparison analysis to show the feasibility, applicability and effectiveness of the proposed strategy.
\end{abstract}

Keywords: neutrosophic set; interval bipolar neutrosophic set; multi-attribute decision making; distance measures; similarity measures

\section{Introduction}

In order to deal with problems involving indefinite and uncertain information, Zhang [1, 2] proposed the bipolar fuzzy sets (BFSs) that utilize the idea of positive and negative preferences of information where the value of membership grade of a component of BFS belongs to [-1, 1]. Later, Ezhilmaran and Shankar [3] proposed the bipolar intuitionistic fuzzy sets (BIFSs). Deli et al. [4] defined the bipolar neutrosophic sets (BNSs) as the generalization of fuzzy sets [5], BFSs [1, 2], intuitionistic fuzzy sets [6], BIFSs [3] and neutrosophic sets [7], etc. In the same research, Deli et al. [4] presented some operations including the score, accuracy, and certainty functions and two weighted operators to develop a bipolar neutrosophic multi-criteria decision making (MCDM) method. Dey et al. [8] investigated a technique for order preference by similarity to ideal solution (TOPSIS) strategy for multi-attribute decision making (MADM) problems in bipolar neutrosophic setting to obtain the best alternative.

Similarity measure is an important decision making device for many MADM problems such as investment [9, 10], pattern recognition [11], supplier selection, construction project selection [12], etc. Deli and Subas [13] and Sahin et al. [14] presented MCDM strategy using correlation coefficient and Jaccard vector similarity measures, respectively. Ulucay et al. [15] proposed four similarity measures 
namely, Dice, weighted Dice, hybrid and weighted hybrid similarity measure of BNSs and applied them to solve MADM problems. Pramanik et al. [9] presented several projections measures such as bidirectional and hybrid projection measures of BNSs and established their basic properties. In the same research, Pramanik et al. [9], developed three novel algorithms for MADM problems in BNS environment.

Deli et al. [16] and Mahmood et al. [17] grounded the concept of interval bipolar neutrosophic sets (IBNSs) by extending the idea of BNSs [4] and interval neutrosophic sets (INSs) [18]. In same paper, Deli et al. [16] defined score, accuracy and certainty functions of IBNS and IBNS weighted average operator and IBNS weighted geometric operator to aggregate the IBNSs and finally, presented a MCDM algorithm. Mahmood et al. [17] defined the union, complement, intersection, and containment of IBNSs and defined some aggregation operators to establish a MADM strategy for IBNSs. In 2018, Pramanik et al. [19] proposed weighted cross entropy measures for bipolar and interval bipolar neutrosophic sets and established their basic properties. Using the weighted cross entropy measures, Pramanik et al. [19] also developed two new MADM strategies. Recently, Pramanik et al. [20] defined correlation coefficient and weighted correlation coefficient measures of IBNSs and established their basic properties and developed a novel MADM strategy with interval bipolar neutrosophic information.

\section{Research gap:}

MADM strategy based on some new similarity measures in interval bipolar neutrosophic set environment.

\section{Motivation:}

The above-mentioned analysis presents the motivation behind proposing new similarity measure based MADM strategies for MADM problems in IBNS environment.

The objectives of the paper are as follows:

1. To define Hamming distance and Euclidean distance measures and prove their basic properties.

2. To introduce a new similarity measure and prove its basic properties.

3. To establish three new MADM strategies based on Hamming distance measure, Euclidean distance measure and a similarity measure using on minimum and maximum operators in IBNS environment.

To deal with the research gap, we propose novel similarity measures for solving MADM problems with interval bipolar neutrosophic information.

The remaining of the article is organized as follows. Section 2 presents some concepts concerned with BFSs, BIFSs, BNSs, and IBNSs. Section 3 introduces new similarity measures based on Hamming and Euclidean distances. Section 3 also introduces new similarity measures between two IBNSs on the basis of maximum and minimum operators and establishes their properties. Section 4 develops three novel MADM strategies in IBNS environment. Section 5 presents algorithmic representation of the developed three MADM strategies in interval bipolar neutrosophic set environment. Section 6 demonstrates an illustrative example to show the applicability and effectiveness of the proposed strategy and then comparison analysis is presented. Finally, some remarks and future scope of research are provided in the concluding section.

\section{Preliminary}


In this section, we recall several basic definitions and properties of BFSs, BIFSs, BNSs, IBNSs respectively, which are useful for the presentation of the paper.

\subsection{Bipolar fuzzy sets}

A BFS [1, 2] $F$ is characterized by a positive membership function $\mu_{F}^{+}(x)$ and a negative membership function $\mu_{F}^{-}(x)$ where $x \in X$. A BFS $F$ is expressed as follows:

$$
F=\left\{x,\left\langle\mu_{F}^{+}(x), \mu_{F}^{-}(x)\right\rangle \mid x \in X\right\}
$$

where $\mu_{F}^{+}(x): X \rightarrow[0,1]$ and $\mu_{F}^{-}(x): X \rightarrow[-1,0]$ for each point $x \in X$.

\subsection{Bipolar intuitionistic fuzzy sets}

Assume that $X$ is a non-empty set, then a BIFS [3] $G$ in $X$ is expressed as:

$$
G=\left\{x,\left\langle\delta_{G}^{+}(x), \delta_{G}^{-}(x), \lambda_{G}^{+}(x), \lambda_{G}^{-}(x)\right\rangle \mid x \in X\right\} .
$$

where $\delta_{G}^{+}(x), \lambda_{G}^{+}(x): X \rightarrow[0,1]$ and $\delta_{G}^{-}(x), \lambda_{G}^{-}(x): X \rightarrow[-1,0]$ for each point $x \in X$ such that $0 \leq \delta_{G}^{+}(x)+\lambda_{G}^{+}(x) \leq 1$ and $-1 \leq \delta_{G}^{-}(x)+\lambda_{G}^{-}(x) \leq 0$.

\subsection{Bipolar neutrosophic set}

A BNS [4] $H$ in $X$ is presented as follows:

$$
H=\left\{x,\left\langle\alpha_{H}^{+}(x), \beta_{H}^{+}(x), \gamma_{H}^{+}(x), \alpha_{H}^{-}(x), \beta_{H}^{-}(x), \gamma_{H}^{-}(x)\right\rangle \mid x \in X\right\}
$$

where $\alpha_{H}^{+}(x), \beta_{H}^{+}(x), \gamma_{H}^{+}(x): X \rightarrow[0,1]$ denote the truth, indeterminate, and falsity membership functions respectively of an object $x \in X$ corresponding to $H . \alpha_{H}^{-}(x), \beta_{H}^{-}(x), \gamma_{H}^{-}(x): X \rightarrow[-1,0]$ signify the truth, indeterminate, and falsity membership functions of an object $x \in X$ to some implicit opposite property associated with $H$.

\subsection{Interval bipolar neutrosophic set}

An IBNS $[16,17] K$ in $X$ is represented as follows:

$$
K=\left\{x,\left\langle\begin{array}{l}
{\left[\inf \alpha_{K}^{+}(x), \sup \alpha_{K}^{+}(x)\right],\left[\inf \beta_{K}^{+}(x), \sup \beta_{K}^{+}(x)\right],\left[\inf \gamma_{K}^{+}(x), \sup \gamma_{K}^{+}(x)\right]} \\
{\left[\inf \alpha_{K}^{-}(x), \sup \alpha_{K}^{-}(x)\right],\left[\inf \beta_{K}^{-}(x), \sup \beta_{K}^{-}(x)\right],\left[\inf \gamma_{K}^{-}(x), \sup \gamma_{K}^{-}(x)\right]}
\end{array}\right\rangle \mid x \in X\right\}
$$

where $K$ is characterized by positive and negative truth-membership $\alpha^{+}(x), \alpha^{-}(x)$; indeterminacy-membership $\beta^{+}(x), \beta^{-}(x)$; falsity-membership $\gamma^{+}(x), \gamma^{-}(x)$ functions respectively. 
$\alpha^{+}(x), \beta^{+}(x), \gamma^{+}(x) \subseteq[0,1] ; \alpha^{-}(x), \beta^{-}(x), \gamma^{-}(x) \subseteq[-1,0]$ for all $x \in X$ with the conditions $0 \leq$ $\sup \alpha^{+}(x)+\sup \beta^{+}(x)+\sup \gamma^{+}(x) \leq 3$, and $-3 \leq \sup \alpha^{-}(x)+\sup \beta^{-}(x)+\sup \gamma^{-}(x) \leq 0$.

Definition 1. Ref. [16, 17]: Consider $L$ and $M$ be two IBNSs in the universe of discourse $X$. We call $L$ is contained in $M$ i.e. $L \subseteq M$ if and only if $\inf \alpha_{L}^{+}(x) \leq \inf \alpha_{M}^{+}(x), \sup \alpha_{L}^{+}(x) \leq \sup \alpha_{M}^{+}(x), \inf \beta_{L}^{+}(x) \geq \inf \beta_{M}^{+}(x), \sup \beta_{L}^{+}(x) \geq \sup \beta_{M}^{+}(x)$, $\inf \gamma_{L}^{+}(x) \geq \inf \gamma_{M}^{+}(x), \sup \gamma_{L}^{+}(x) \geq \sup \gamma_{M}^{+}(x), \inf \alpha_{L}^{-}(x) \geq \inf \alpha_{M}^{-}(x), \sup \alpha_{L}^{-}(x) \geq \sup \alpha_{M}^{-}(x)$, $\inf \beta_{L}^{-}(x) \leq \inf \beta_{M}^{-}(x), \sup \beta_{L}^{-}(x) \leq \sup \beta_{M}^{-}(x), \inf \alpha_{L}^{-}(x) \leq \inf \alpha_{M}^{-}(x), \sup \gamma_{L}^{-}(x) \leq \sup \gamma_{M}^{-}(x)$, for all $x \in X$.

Definition 2. Ref. [16, 17]: Suppose $L$ and $M$ are two IBNSs defined over $X$. Then we say $L=M$ if and only if $\inf \alpha_{L}^{+}(x)=\inf \alpha_{M}^{+}(x), \sup \alpha_{L}^{+}(x)=\sup \alpha_{M}^{+}(x), \inf \beta_{L}^{+}(x)=\inf \beta_{M}^{+}(x), \sup \beta_{L}^{+}(x)=\sup \beta_{M}^{+}(x), \inf \gamma_{L}^{+}(x)=$ $\inf \gamma_{M}^{+}(x), \sup \gamma_{L}^{+}(x)=\sup \gamma_{M}^{+}(x), \inf \alpha_{L}^{-}(x)=\inf \alpha_{M}^{-}(x), \sup \alpha_{L}^{-}(x)=\sup \alpha_{M}^{-}(x), \inf \beta_{L}^{-}(x)=\inf \beta_{M}^{-}(x)$, $\sup \beta_{L}^{-}(x)=\sup \beta_{M}^{-}(x), \inf \alpha_{L}^{-}(x)=\inf \alpha_{M}^{-}(x), \sup \gamma_{L}^{-}(x)=\sup \gamma_{M}^{-}(x)$, for all $x \in X$.

Definition 3. Ref. [18]: The complement of an IBNS $L=\left\{x,<\left[\inf \alpha_{L}^{+}(x), \sup \alpha_{L}^{+}(x)\right] ; \inf \beta_{L}^{+}(x)\right.$, $\left.\sup \beta_{L}^{+}(x)\right] ;\left[\inf \gamma_{L}^{+}(x), \sup \gamma_{L}^{+}(x)\right] ;\left[\inf \alpha_{L}^{-}(x), \sup \alpha_{L}^{-}(x)\right] ;\left[\inf \beta_{L}^{-}(x), \sup \beta_{L}^{-}(x)\right] ;\left[\inf \gamma_{L}^{-}(x), \sup \gamma_{L}^{-}(x)\right]>$ $\mid x \in X\}$ is represented as $L^{c}=\left\{x,<\left[\inf \alpha_{L^{c}}^{+}(x), \sup \alpha_{L^{c}}^{+}(x)\right] ;\left[\inf \beta_{L^{c}}^{+}(x), \sup \beta_{L^{c}}^{+}(x)\right] ;\left[\inf \gamma_{L^{c}}^{+}(x)\right.\right.$, $\left.\left.\sup \gamma_{L^{c}}^{+}(x)\right] ;\left[\inf \alpha_{L^{c}}^{-}(x), \sup \alpha_{L^{c}}^{-}(x)\right] ;\left[\inf \beta_{L^{c}}^{-}(x), \sup \beta_{L^{c}}^{-}(x)\right] ;\left[\inf \gamma_{L^{c}}^{-}(x), \sup \gamma_{L^{c}}^{-}(x)\right]>\mid x \in X\right\}$ where $\inf \alpha_{L^{c}}^{+}(x)=\inf \gamma_{L}^{+}(x), \sup \alpha_{L^{c}}^{+}(x)=\sup \gamma_{L}^{+}(x), \inf \beta_{L^{c}}^{+}(x)=1-\sup \beta_{L}^{+}(x), \sup \beta_{L^{c}}^{+}(x)=1-\inf \beta_{L}^{+}(x)$, $\inf \gamma_{L^{c}}^{+}(x)=\inf \alpha_{L}^{+}, \sup \gamma_{L^{c}}^{+}(x)=\sup \alpha_{L}^{+}(x), \inf \alpha_{L^{c}}^{-}(x)=\inf \gamma_{L}^{-}(x), \sup \alpha_{L^{c}}^{-}(x)=\sup \gamma_{L}^{-}(x), \inf \beta_{L^{c}}^{-}(x)=-1$ $-\sup \beta_{L}^{-}(x), \sup \beta_{L^{c}}^{-}(x)=-1-\inf \beta_{L}^{-}(x), \inf \gamma_{L^{c}}^{-}(x)=\inf \alpha_{L}^{-}(x), \sup \gamma_{L^{c}}^{-}(x)=\sup \alpha_{L}^{-}(x)$ for all $x \in X$.

\section{Similarity measures between IBNSs}

Definition 4: Let two IBNSs $L$ and $M$ in $X=\left\{x_{1}, x_{2}, \ldots, x_{\mathrm{m}}\right\}$ be represented by $L=\left\{x_{\mathrm{i}},\left[\inf \alpha_{L}^{+}\left(x_{\mathrm{i}}\right)\right.\right.$, $\left.\sup \alpha_{L}^{+}\left(x_{\mathrm{i}}\right)\right],\left[\inf \beta_{L}^{+}\left(x_{\mathrm{i}}\right), \sup \beta_{L}^{+}\left(x_{\mathrm{i}}\right)\right],\left[\inf \gamma_{L}^{+}\left(x_{\mathrm{i}}\right), \sup \gamma_{L}^{+}\left(x_{\mathrm{i}}\right)\right],\left[\inf \alpha_{L}^{-}\left(x_{\mathrm{i}}\right), \sup \alpha_{L}^{-}\left(x_{\mathrm{i}}\right)\right],\left[\inf \beta_{L}^{-}\left(x_{\mathrm{i}}\right)\right.$, $\left.\left.\sup \beta_{L}^{-}\left(x_{\mathrm{i}}\right)\right],\left[\inf \gamma_{L}^{-}\left(x_{\mathrm{i}}\right), \sup \gamma_{L}^{-}\left(x_{\mathrm{i}}\right)\right] \mid x \in X\right\}$ and $M=\left\{x_{\mathrm{i}}, \quad\left[\inf \alpha_{M}^{+}\left(x_{\mathrm{i}}\right), \sup \alpha_{M}^{+}\left(x_{\mathrm{i}}\right)\right], \quad\left[\inf \beta_{M}^{+}\left(x_{\mathrm{i}}\right)\right.\right.$, 
$\left.\sup \beta_{M}^{+}\left(x_{\mathrm{i}}\right)\right],\left[\inf \gamma_{M}^{+}\left(x_{\mathrm{i}}\right), \sup \gamma_{M}^{+}\left(x_{\mathrm{i}}\right)\right],\left[\inf \alpha_{M}^{-}\left(x_{\mathrm{i}}\right), \sup \alpha_{M}^{-}\left(x_{\mathrm{i}}\right)\right],\left[\inf \beta_{M}^{-}\left(x_{\mathrm{i}}\right), \sup \beta_{M}^{-}\left(x_{\mathrm{i}}\right)\right],\left[\inf \gamma_{M}^{-}\left(x_{\mathrm{i}}\right)\right.$, $\left.\left.\sup \gamma_{M}^{-}\left(x_{\mathrm{i}}\right)\right] \mid x_{\mathrm{i}} \in X\right\}$, where $0 \leq \sup \alpha_{L}^{+}(x)+\sup \beta_{L}^{+}(x)+\sup \gamma_{L}^{+}(x) \leq 3 ;-3 \leq \sup \alpha_{L}^{-}(x)+\sup \beta_{L}^{-}(x)+$ $\sup \gamma_{L}^{-}(x) \leq 0 ; 0 \leq \sup \alpha_{M}^{+}(x)+\sup \beta_{M}^{+}(x)+\sup \gamma_{M}^{+}(x) \leq 3 ;$ and $-3 \leq \sup \alpha_{M}^{-}(x)+\sup \beta_{M}^{-}(x)+\sup \gamma_{M}^{-}$ $(x) \leq 0$. Then, we define the Hamming and Euclidean distances between $L$ and $M$ as follows:

(i). The Hamming distance

$D_{1}(L, M)=\frac{1}{12} \sum_{j=1}^{m}\left(\begin{array}{l}\left|\inf \alpha_{L}^{+}\left(x_{j}\right)-\inf \alpha_{M}^{+}\left(x_{j}\right)\right|+\left|\sup \alpha_{L}^{+}\left(x_{j}\right)-\sup \alpha_{M}^{+}\left(x_{j}\right)\right|+\left|\inf \beta_{L}^{+}\left(x_{j}\right)-\inf \beta_{M}^{+}\left(x_{j}\right)\right|+ \\ \left|\sup \beta_{L}^{+}\left(x_{j}\right)-\sup \beta_{M}^{+}\left(x_{j}\right)\right|+\left|\inf \gamma_{L}^{+}\left(x_{j}\right)-\inf \gamma_{M}^{+}\left(x_{j}\right)\right|+\left|\sup \gamma_{L}^{+}\left(x_{j}\right)-\sup \gamma_{M}^{+}\left(x_{j}\right)\right|+ \\ \left|\inf \alpha_{L}^{-}\left(x_{j}\right)-\inf \alpha_{M}^{-}\left(x_{j}\right)\right|+\left|\sup \alpha_{L}^{-}\left(x_{j}\right)-\sup \alpha_{M}^{-}\left(x_{j}\right)\right|+\left|\inf \beta_{L}^{-}\left(x_{j}\right)-\inf \beta_{M}^{-}\left(x_{j}\right)\right|+ \\ \left|\sup \beta_{L}^{-}\left(x_{j}\right)-\sup \beta_{M}^{-}\left(x_{j}\right)\right|+\left|\inf \gamma_{L}^{-}\left(x_{j}\right)-\inf \gamma_{M}^{-}\left(x_{j}\right)\right|+\left|\sup \gamma_{L}^{-}\left(x_{j}\right)-\sup \gamma_{M}^{-}\left(x_{j}\right)\right|\end{array}\right)$

(ii) The normalized Hamming distance

$D_{2}(L, M)=\frac{1}{12 m} m_{j=1}^{m}\left(\begin{array}{l}\left|\inf \alpha_{L}^{+}\left(x_{j}\right)-\inf \alpha_{M}^{+}\left(x_{j}\right)\right|+\left|\sup \alpha_{L}^{+}\left(x_{j}\right)-\sup \alpha_{M}^{+}\left(x_{j}\right)\right|+\left|\inf \beta_{L}^{+}\left(x_{j}\right)-\inf \beta_{M}^{+}\left(x_{j}\right)\right|+ \\ \left|\sup \beta_{L}^{+}\left(x_{j}\right)-\sup \beta_{M}^{+}\left(x_{j}\right)\right|+\left|\inf \gamma_{L}^{+}\left(x_{j}\right)-\inf \gamma_{M}^{+}\left(x_{j}\right)\right|+\left|\sup \gamma_{L}^{+}\left(x_{j}\right)-\sup \gamma_{M}^{+}\left(x_{j}\right)\right|+ \\ \left|\inf \alpha_{L}^{-}\left(x_{j}\right)-\inf \alpha_{M}^{-}\left(x_{j}\right)\right|+\left|\sup \alpha_{L}^{-}\left(x_{j}\right)-\sup \alpha_{M}^{-}\left(x_{j}\right)\right|+\left|\inf \beta_{L}^{-}\left(x_{j}\right)-\inf \beta_{M}^{-}\left(x_{j}\right)\right|+ \\ \sup \beta_{L}^{-}\left(x_{j}\right)-\sup \beta_{M}^{-}\left(x_{j}\right)|+| \inf \gamma_{L}^{-}\left(x_{j}\right)-\inf \gamma_{M}^{-}\left(x_{j}\right)|+| \sup \gamma_{L}^{-}\left(x_{j}\right)-\sup \gamma_{M}^{-}\left(x_{j}\right) \mid\end{array}\right)$

(iii). The Euclidean distance

$D_{3}(L, M)=\left\{\begin{array}{l}\frac{1}{12} \sum_{j=1}^{m}\left[\left(\inf \alpha_{L}^{+}\left(x_{j}\right)-\inf \alpha_{M}^{+}\left(x_{j}\right)\right)^{2}+\left(\sup \alpha_{L}^{+}\left(x_{j}\right)-\sup \alpha_{M}^{+}\left(x_{j}\right)\right)^{2}+\left(\inf \beta_{L}^{+}\left(x_{j}\right)-\inf \beta_{M}^{+}\left(x_{j}\right)\right)^{2}+\right. \\ \left(\sup \beta_{L}^{+}\left(x_{j}\right)-\sup \beta_{M}^{+}\left(x_{j}\right)\right)^{2}+\left(\inf \gamma_{L}^{+}\left(x_{j}\right)-\inf \gamma_{M}^{+}\left(x_{j}\right)\right)^{2}+\left(\sup \gamma_{L}^{+}\left(x_{j}\right)-\sup \gamma_{M}^{+}\left(x_{j}\right)\right)^{2}+ \\ \left(\inf \alpha_{L}^{-}\left(x_{j}\right)-\inf \alpha_{M}^{-}\left(x_{j}\right)\right)^{2}+\left(\sup \alpha_{L}^{-}\left(x_{j}\right)-\sup \alpha_{M}^{-}\left(x_{j}\right)\right)^{2}+\left(\inf \beta_{L}^{-}\left(x_{j}\right)-\inf \beta_{M}^{-}\left(x_{j}\right)\right)^{2}+ \\ \left.\left(\sup \beta_{L}^{-}\left(x_{j}\right)-\sup \beta_{M}^{-}\left(x_{j}\right)\right)^{2}+\left(\inf \gamma_{L}^{-}\left(x_{j}\right)-\inf \gamma_{M}^{-}\left(x_{j}\right)\right)^{2}+\left(\sup \gamma_{L}^{-}\left(x_{j}\right)-\sup \gamma_{M}^{-}\left(x_{j}\right)\right)^{2}\right]\end{array}\right\}$

(iv). The normalized Euclidean distance

$D_{4}(L, M)=\left\{\begin{array}{l}\frac{1}{12 m} \sum_{j=1}^{m}\left[\left(\inf \alpha_{L}^{+}\left(x_{j}\right)-\inf \alpha_{M}^{+}\left(x_{j}\right)\right)^{2}+\left(\sup \alpha_{L}^{+}\left(x_{j}\right)-\sup \alpha_{M}^{+}\left(x_{j}\right)\right)^{2}+\left(\inf \beta_{L}^{+}\left(x_{j}\right)-\inf \beta_{M}^{+}\left(x_{j}\right)\right)^{2}+\right. \\ \left(\sup \beta_{L}^{+}\left(x_{j}\right)-\sup \beta_{M}^{+}\left(x_{j}\right)\right)^{2}+\left(\inf \gamma_{L}^{+}\left(x_{j}\right)-\inf \gamma_{M}^{+}\left(x_{j}\right)\right)^{2}+\left(\sup \gamma_{L}^{+}\left(x_{j}\right)-\sup \gamma_{M}^{+}\left(x_{j}\right)\right)^{2}+ \\ \left(\inf \alpha_{L}^{-}\left(x_{j}\right)-\inf \alpha_{M}^{-}\left(x_{j}\right)\right)^{2}+\left(\sup \alpha_{L}^{-}\left(x_{j}\right)-\sup \alpha_{M}^{-}\left(x_{j}\right)\right)^{2}+\left(\inf \beta_{L}^{-}\left(x_{j}\right)-\inf \beta_{M}^{-}\left(x_{j}\right)\right)^{2}+ \\ \left.\left(\sup \beta_{L}^{-}\left(x_{j}\right)-\sup \beta_{M}^{-}\left(x_{j}\right)\right)^{2}+\left(\inf \gamma_{L}^{-}\left(x_{j}\right)-\inf \gamma_{M}^{-}\left(x_{j}\right)\right)^{2}+\left(\sup \gamma_{L}^{-}\left(x_{j}\right)-\sup \gamma_{M}^{-}\left(x_{j}\right)\right)^{2}\right]\end{array}\right\}$

However, if we consider $w_{j}=\left(w_{1}, w_{2}, \ldots, w_{m}\right)$ be the weight vector of the objects $x_{j}(j=1,2, \ldots, m)$, then, the weighted Hamming and weighted Euclidean distance measures are defined as follows:

$$
\begin{aligned}
& D_{5}(L, M)=\frac{1}{12} \sum_{j=1}^{m} w_{j}\left(\begin{array}{l}
\left|\inf \alpha_{L}^{+}\left(x_{j}\right)-\inf \alpha_{M}^{+}\left(x_{j}\right)\right|+\left|\sup \alpha_{L}^{+}\left(x_{j}\right)-\sup \alpha_{M}^{+}\left(x_{j}\right)\right|+\left|\inf \beta_{L}^{+}\left(x_{j}\right)-\inf \beta_{M}^{+}\left(x_{j}\right)\right|+ \\
\left|\sup \beta_{L}^{+}\left(x_{j}\right)-\sup \beta_{M}^{+}\left(x_{j}\right)\right|+\left|\inf \gamma_{L}^{+}\left(x_{j}\right)-\inf \gamma_{M}^{+}\left(x_{j}\right)\right|+\left|\sup \gamma_{L}^{+}\left(x_{j}\right)-\sup \gamma_{M}^{+}\left(x_{j}\right)\right|+ \\
\inf \alpha_{L}^{-}\left(x_{j}\right)-\inf \alpha_{M}^{-}\left(x_{j}\right)|+| \sup \alpha_{L}^{-}\left(x_{j}\right)-\sup \alpha_{M}^{-}\left(x_{j}\right)|+| \inf \beta_{L}^{-}\left(x_{j}\right)-\inf \beta_{M}^{-}\left(x_{j}\right) \mid+ \\
\left|\sup \beta_{L}^{-}\left(x_{j}\right)-\sup \beta_{M}^{-}\left(x_{j}\right)\right|+\left|\inf \gamma_{L}^{-}\left(x_{j}\right)-\inf \gamma_{M}^{-}\left(x_{j}\right)\right|+\left|\sup \gamma_{L}^{-}\left(x_{j}\right)-\sup \gamma_{M}^{-}\left(x_{j}\right)\right|
\end{array}\right) \\
& D_{6}(L, M)=\left\{\begin{array}{l}
\frac{1}{12} \sum_{j=1}^{m} w_{j}\left[\left(\inf \alpha_{L}^{+}\left(x_{j}\right)-\inf \alpha_{M}^{+}\left(x_{j}\right)\right)^{2}+\left(\sup \alpha_{L}^{+}\left(x_{j}\right)-\sup \alpha_{M}^{+}\left(x_{j}\right)\right)^{2}+\left(\inf \beta_{L}^{+}\left(x_{j}\right)-\inf \beta_{M}^{+}\left(x_{j}\right)\right)^{2}+\right. \\
\left(\sup \beta_{L}^{+}\left(x_{j}\right)-\sup \beta_{M}^{+}\left(x_{j}\right)\right)^{2}+\left(\inf \gamma_{L}^{+}\left(x_{j}\right)-\inf \gamma_{M}^{+}\left(x_{j}\right)\right)^{2}+\left(\sup \gamma_{L}^{+}\left(x_{j}\right)-\sup \gamma_{M}^{+}\left(x_{j}\right)\right)^{2}+ \\
\left(\inf \alpha_{L}^{-}\left(x_{j}\right)-\inf \alpha_{M}^{-}\left(x_{j}\right)\right)^{2}+\left(\sup \alpha_{L}^{-}\left(x_{j}\right)-\sup \alpha_{M}^{-}\left(x_{j}\right)\right)^{2}+\left(\inf \beta_{L}^{-}\left(x_{j}\right)-\inf \beta_{M}^{-}\left(x_{j}\right)\right)^{2}+ \\
\left.\left(\sup \beta_{L}^{-}\left(x_{j}\right)-\sup \beta_{M}^{-}\left(x_{j}\right)\right)^{2}+\left(\inf \gamma_{L}^{-}\left(x_{j}\right)-\inf \gamma_{M}^{-}\left(x_{j}\right)\right)^{2}+\left(\sup \gamma_{L}^{-}\left(x_{j}\right)-\sup \gamma_{M}^{-}\left(x_{j}\right)\right)^{2}\right]
\end{array}\right\}
\end{aligned}
$$

If we take $w=(1 / m, 1 / m, \ldots, 1 / m)$, the Eqs. (5) and (6) are reduced to Hamming and Euclidean distances, respectively. 
6 of 18

Theorem 1. The above defined distance measures $D_{l}(L, M)(l=1,2,3,4,5,6)$ between $L, M$ satisfies the following properties:

(P1) $0 \leq D_{l}(L, M) \leq 1,(l=1,2,3,4,5,6)$

(P2) $D_{l}(L, M)=0$, if and only if $L=M,(l=1,2,3,4,5,6)$

(P3) $D_{l}(L, M)=D_{l}(M, L),(l=1,2,3,4,5,6)$

(P4) If $L \subseteq M \subseteq N, N$ is an IBNS in $X$, then $D_{l}(L, M) \leq D_{l}(L, N)$ and $D_{l}(M, N) \leq D_{l}(L, N),(l=1,2$, $3,4,5,6)$.

Proof

(P1) Clearly, from the definitions, we have $0 \leq D_{l}(L, M) \leq 1,(l=1,2,3,4,5,6)$.

$\left(\mathrm{P}_{2}\right)$ If inf $\alpha_{L}^{+}(x)=\inf \alpha_{M}^{+}(x), \sup \alpha_{L}^{+}(x)=\sup \alpha_{M}^{+}(x), \inf \beta_{L}^{+}(x)=\inf \beta_{M}^{+}(x), \sup \beta_{L}^{+}(x)=\sup \beta_{M}^{+}(x)$, $\inf \gamma_{L}^{+}(x)=\inf \gamma_{M}^{+}(x), \sup \gamma_{L}^{+}(x)=\sup \gamma_{M}^{+}(x), \inf \alpha_{L}^{-}(x)=\inf \alpha_{M}^{-}(x), \sup \alpha_{L}^{-}(x)=\sup \alpha_{M}^{-}(x), \inf \beta_{L}^{-}(x)=$ $\inf \beta_{M}^{-}(x), \sup \beta_{L}^{-}(x)=\sup \beta_{M}^{-}(x), \inf \alpha_{L}^{-}(x)=\inf \alpha_{M}^{-}(x), \sup \gamma_{L}^{-}(x)=\sup \gamma_{M}^{-}(x)$, for all $x \in X$, then $D_{l}(L$, $M)=0,(l=1,2,3,4,5,6)$ and conversely.

$\left(\mathrm{P}_{3}\right)$ From the above definitions $(1-6)$, we have $D_{l}(L, M)=D_{l}(M, L),(l=1,2,3,4,5,6)$.

( $\left.\mathrm{P}_{4}\right)$ Consider, $L \subseteq M \subseteq N$, then we have inf $\alpha_{L}^{+}(x) \leq \inf \alpha_{M}^{+}(x) \leq \inf \alpha_{N}^{+}(x)$, $\sup \alpha_{L}^{+}(x) \leq \sup \alpha_{M}^{+}(x) \leq \sup \alpha_{N}^{+}(x), \inf \beta_{L}^{+}(x) \geq \inf \beta_{M}^{+}(x) \geq \inf \beta_{N}^{+}(x)$, $\sup \beta_{L}^{+}(x) \geq \sup \beta_{M}^{+}(x) \geq \sup \beta_{N}^{+}(x), \inf \gamma_{L}^{+}(x) \geq \inf \gamma_{M}^{+}(x) \geq \inf \gamma_{N}^{+}(x), \sup \gamma_{L}^{+}(x) \geq \sup \gamma_{M}^{+}(x) \geq \sup \gamma_{N}^{+}(x)$, $\inf \alpha_{L}^{-}(x) \geq \inf \alpha_{M}^{-}(x) \geq \inf \alpha_{N}^{-}(x), \sup \alpha_{L}^{-}(x) \geq \sup \alpha_{M}^{-}(x) \geq \sup \alpha_{N}^{-}(x), \inf \beta_{L}^{-}(x) \leq \inf \beta_{M}^{-}(x) \leq \inf \beta_{N}^{-}(x)$, $\sup \beta_{L}^{-}(x) \leq \sup \beta_{M}^{-}(x) \leq \sup \beta_{N}^{-}(x)$, inf $\gamma_{L}^{-}(x) \leq \inf \gamma_{M}^{-}(x) \leq \inf \gamma_{N}^{-}(x), \sup \gamma_{L}^{-}(x) \leq \sup \gamma_{M}^{-}(x)$ $\leq \sup \gamma_{N}^{-}(x)$ for all $x \in X$. For $\mathrm{r}=1,2$, we have

$\left|\inf \alpha_{L}^{+}\left(x_{j}\right)-\inf \alpha_{M}^{+}\left(x_{j}\right)\right| \mathrm{r} \leq\left|\inf \alpha_{L}^{+}\left(x_{j}\right)-\inf \alpha_{N}^{+}\left(x_{j}\right)\right| \mathrm{r},\left|\sup \alpha_{L}^{+}\left(x_{j}\right)-\sup \alpha_{M}^{+}\left(x_{j}\right)\right| \mathrm{r} \leq \mid \sup \alpha_{L}^{+}\left(x_{j}\right)-$ $\sup \alpha_{N}^{+}\left(x_{j}\right)|\mathrm{r},| \inf \beta_{L}^{+}\left(x_{j}\right)-\inf \beta_{M}^{+}\left(x_{j}\right)|\mathrm{r} \leq| \inf \beta_{L}^{+}\left(x_{j}\right)-\inf \beta_{N}^{+}\left(x_{j}\right)|\mathrm{r},| \sup \beta_{L}^{+}\left(x_{j}\right)-\sup \beta_{M}^{+}\left(x_{j}\right)|\mathrm{r} \leq|$ $\sup \beta_{L}^{+}\left(x_{j}\right)-\sup \beta_{N}^{+}\left(x_{j}\right)|\mathrm{r},| \inf \gamma_{L}^{+}\left(x_{j}\right)-\inf \gamma_{M}^{+}\left(x_{j}\right)|\mathrm{r} \leq| \inf \gamma_{L}^{+}\left(x_{j}\right)-\inf \gamma_{N}^{+}\left(x_{j}\right)|\mathrm{r},| \sup \gamma_{L}^{+}\left(x_{j}\right)-$ $\sup \gamma_{M}^{+}\left(x_{j}\right)|\mathrm{r} \leq| \sup \gamma_{L}^{+}\left(x_{j}\right)-\sup \gamma_{N}^{+}\left(x_{j}\right)|\mathrm{r},| \inf \alpha_{L}^{-}\left(x_{j}\right)-\inf \alpha_{M}^{-}\left(x_{j}\right)|\mathrm{r} \leq| \inf \alpha_{L}^{-}\left(x_{j}\right)-\inf \alpha_{N}^{-}\left(x_{j}\right)|\mathrm{r}$, $\sup \alpha_{L}^{-}\left(x_{j}\right)-\sup \alpha_{M}^{-}\left(x_{j}\right)|\mathrm{r} \leq| \sup \alpha_{L}^{-}\left(x_{j}\right)-\sup \alpha_{N}^{-}\left(x_{j}\right)|\mathrm{r},| \inf \beta_{L}^{-}\left(x_{j}\right)-\inf \beta_{M}^{-}\left(x_{j}\right)|\mathrm{r} \leq| \inf \beta_{L}^{-}\left(x_{j}\right)-$ $\inf \beta_{N}^{-}\left(x_{j}\right)|\mathrm{r},| \sup \beta_{L}^{-}\left(x_{j}\right)-\sup \beta_{M}^{-}\left(x_{j}\right)|\mathrm{r} \leq| \sup \beta_{L}^{-}\left(x_{j}\right)-\sup \beta_{N}^{-}\left(x_{j}\right)|\mathrm{r},| \inf \gamma_{L}^{-}\left(x_{j}\right)-$ $\inf \gamma_{M}^{-}\left(x_{j}\right)|\mathrm{r} \leq| \inf \gamma_{L}^{-}\left(x_{j}\right)-\inf \gamma_{N}^{-}\left(x_{j}\right)|\mathrm{r},| \sup \gamma_{L}^{-}\left(x_{j}\right)-\sup \gamma_{M}^{-}\left(x_{j}\right)|\mathrm{r} \leq| \sup \gamma_{L}^{-}\left(x_{j}\right)-\sup \gamma_{N}^{-}\left(x_{j}\right) \mid \mathrm{r}$ 
By adding the above inequalities, we have $D_{l}(L, M) \leq D_{l}(L, N), l=1,2, \ldots, 6$. Again, we have, $\left|\inf \alpha_{M}^{+}\left(x_{j}\right)-\inf \alpha_{N}^{+}\left(x_{j}\right)\right| \mathrm{r} \leq\left|\inf \alpha_{L}^{+}\left(x_{j}\right)-\inf \alpha_{N}^{+}\left(x_{j}\right)\right| \mathrm{r},\left|\sup \alpha_{M}^{+}\left(x_{j}\right)-\sup \alpha_{N}^{+}\left(x_{j}\right)\right| \mathrm{r} \leq \mid \sup \alpha_{L}^{+}\left(x_{j}\right)-$ $\sup \alpha_{N}^{+}\left(x_{j}\right)|\mathrm{r},| \inf \beta_{M}^{+}\left(x_{j}\right)-\inf \beta_{N}^{+}\left(x_{j}\right)|\mathrm{r} \leq| \inf \beta_{L}^{+}\left(x_{j}\right)-\inf \beta_{N}^{+}\left(x_{j}\right)|\mathrm{r},| \sup \beta_{M}^{+}\left(x_{j}\right)-$ $\sup \beta_{N}^{+}\left(x_{j}\right)|\mathrm{r} \leq| \sup \beta_{L}^{+}\left(x_{j}\right)-\sup \beta_{N}^{+}\left(x_{j}\right)|\mathrm{r},| \inf \gamma_{M}^{+}\left(x_{j}\right)-\inf \gamma_{N}^{+}\left(x_{j}\right)|\mathrm{r} \leq| \inf \gamma_{L}^{+}\left(x_{j}\right)-\inf \gamma_{N}^{+}\left(x_{j}\right)|\mathrm{r}$, $\sup \gamma_{M}^{+}\left(x_{j}\right)-\sup \gamma_{N}^{+}\left(x_{j}\right)|\mathrm{r} \leq| \sup \gamma_{L}^{+}\left(x_{j}\right)-\sup \gamma_{N}^{+}\left(x_{j}\right)|\mathrm{r},| \inf \alpha_{M}^{-}\left(x_{j}\right)-\inf \alpha_{N}^{-}\left(x_{j}\right)|\mathrm{r} \leq| \inf \alpha_{L}^{-}\left(x_{j}\right)-$ $\inf \alpha_{N}^{-}\left(x_{j}\right)|\mathrm{r},| \sup \alpha_{M}^{-}\left(x_{j}\right)-\sup \alpha_{N}^{-}\left(x_{j}\right)|\mathrm{r} \leq| \sup \alpha_{L}^{-}\left(x_{j}\right)-\sup \alpha_{N}^{-}\left(x_{j}\right)|\mathrm{r},| \inf \beta_{M}^{-}\left(x_{j}\right)-$ $\inf \beta_{N}^{-}\left(x_{j}\right)|\mathrm{r} \leq| \inf \beta_{L}^{-}\left(x_{j}\right)-\inf \beta_{N}^{-}\left(x_{j}\right)|\mathrm{r},| \sup \beta_{M}^{-}\left(x_{j}\right)-\sup \beta_{N}^{-}\left(x_{j}\right)|\mathrm{r} \leq| \sup \beta_{L}^{-}\left(x_{j}\right)-\sup \beta_{N}^{-}\left(x_{j}\right) \mid \mathrm{r}$, $\left|\inf \gamma_{M}^{-}\left(x_{j}\right)-\inf \gamma_{N}^{-}\left(x_{j}\right)\right| \mathrm{r} \leq\left|\inf \gamma_{L}^{-}\left(x_{j}\right)-\inf \gamma_{N}^{-}\left(x_{j}\right)\right| \mathrm{r},\left|\sup \gamma_{M}^{-}\left(x_{j}\right)-\sup \gamma_{N}^{-}\left(x_{j}\right)\right| \mathrm{r} \leq \mid \sup \gamma_{L}^{-}\left(x_{j}\right)-$ $\sup \gamma_{N}^{-}\left(x_{j}\right) \mid \mathrm{r}$, by adding the above inequalities, we get $D_{l}(M, N) \leq D_{l}(L, N), l=1,2, \ldots, 6$.

Example 1. Assume that $L=<[0.2,0.5],[0.6,0.8],[0.5,0.8],[-0.6,-0.3],[-0.7,-0.5],[-0.8,-0.3]>$ and $M=<[0.3,0.5],[0.2,0.5],[0.3,0.7],[-0.6,-0.1],[-0.7,-0.2],[-0.9,-0.1]>$ be two IBNSs, then we determine $D_{1}(L, M)$ and $D_{3}(L, M)$ as follows.

$$
\begin{aligned}
& D_{1}(L, M)=\frac{1}{12}\left\langle\begin{array}{l}
|0.2-0.3|+|0.5-0.5|+|0.6-0.2|+|0.8-0.5|+|0.5-0.3|+|0.8-0.7|+|(-0.6)-(-0.6)|+ \\
|(-0.3)-(-0.1)|+|(-0.7)-(-0.7)|+|(-0.5)-(-0.2)|+|(-0.8)-(-0.9)|+|(-0.3)-(-0.1)|
\end{array}\right\rangle \\
& =0.1583
\end{aligned}
$$

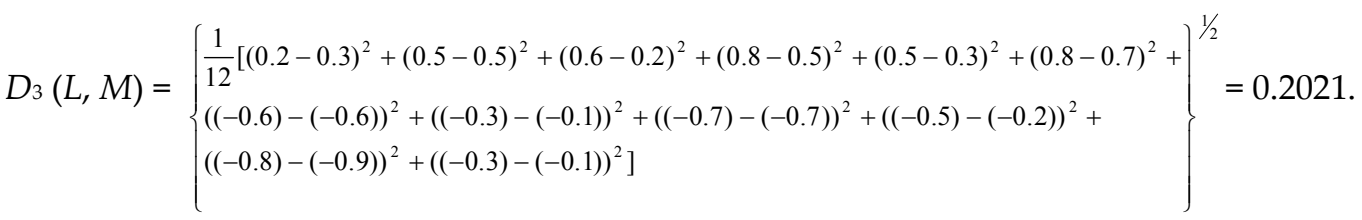

Definition 5: Let $L$ and $M$ be two IBNSs in $X=\left\{x_{1}, x_{2}, \ldots, x_{n}\right\}$, then we propose two similarity measures between $L$ and $M$ based on the defined Hamming and Euclidean distance measures as given below.

$S M_{1}(L, M)=1-\frac{1}{12} \sum_{j=1}^{n} w_{\mathrm{j}}\left(\begin{array}{l}\left|\inf \alpha_{L}^{+}\left(x_{j}\right)-\inf \alpha_{M}^{+}\left(x_{j}\right)\right|+\left|\sup \alpha_{L}^{+}\left(x_{j}\right)-\sup \alpha_{M}^{+}\left(x_{j}\right)\right|+\left|\inf \beta_{L}^{+}\left(x_{j}\right)-\inf \beta_{M}^{+}\left(x_{j}\right)\right|+ \\ \left|\sup \beta_{L}^{+}\left(x_{j}\right)-\sup \beta_{M}^{+}\left(x_{j}\right)\right|+\left|\inf \gamma_{L}^{+}\left(x_{j}\right)-\inf \gamma_{M}^{+}\left(x_{j}\right)\right|+\left|\sup \gamma_{L}^{+}\left(x_{j}\right)-\sup \gamma_{M}^{+}\left(x_{j}\right)\right|+ \\ \left|\inf \alpha_{L}^{-}\left(x_{j}\right)-\inf \alpha_{M}^{-}\left(x_{j}\right)\right|+\left|\sup \alpha_{L}^{-}\left(x_{j}\right)-\sup \alpha_{M}^{-}\left(x_{j}\right)\right|+\left|\inf \beta_{L}^{-}\left(x_{j}\right)-\inf \beta_{M}^{-}\left(x_{j}\right)\right|+ \\ \left|\sup \beta_{L}^{-}\left(x_{j}\right)-\sup \beta_{M}^{-}\left(x_{j}\right)\right|+\left|\inf \gamma_{L}^{-}\left(x_{j}\right)-\inf \gamma_{M}^{-}\left(x_{j}\right)\right|+\left|\sup \gamma_{L}^{-}\left(x_{j}\right)-\sup \gamma_{M}^{-}\left(x_{j}\right)\right|\end{array}\right)$

$S M_{2}(L, M)=1-\left\{\begin{array}{l}\frac{1}{12} \sum_{j=1}^{n} w_{\mathrm{j}}\left[\left(\inf \alpha_{L}^{+}\left(x_{j}\right)-\inf \alpha_{M}^{+}\left(x_{j}\right)\right)^{2}+\left(\sup \alpha_{L}^{+}\left(x_{j}\right)-\sup \alpha_{M}^{+}\left(x_{j}\right)\right)^{2}+\left(\inf \beta_{L}^{+}\left(x_{j}\right)-\inf \beta_{M}^{+}\left(x_{j}\right)\right)^{2}+\right. \\ \left(\sup \beta_{L}^{+}\left(x_{j}\right)-\sup \beta_{M}^{+}\left(x_{j}\right)\right)^{2}+\left(\inf \gamma_{L}^{+}\left(x_{j}\right)-\inf \gamma_{M}^{+}\left(x_{j}\right)\right)^{2}+\left(\sup \gamma_{L}^{+}\left(x_{j}\right)-\sup \gamma_{M}^{+}\left(x_{j}\right)\right)^{2}+ \\ \left(\inf \alpha_{L}^{-}\left(x_{j}\right)-\inf \alpha_{M}^{-}\left(x_{j}\right)\right)^{2}+\left(\sup \alpha_{L}^{-}\left(x_{j}\right)-\sup \alpha_{M}^{-}\left(x_{j}\right)\right)^{2}+\left(\inf \beta_{L}^{-}\left(x_{j}\right)-\inf \beta_{M}^{-}\left(x_{j}\right)\right)^{2}+ \\ \left.\left(\sup \beta_{L}^{-}\left(x_{j}\right)-\sup \beta_{M}^{-}\left(x_{j}\right)\right)^{2}+\left(\inf \gamma_{L}^{-}\left(x_{j}\right)-\inf \gamma_{M}^{-}\left(x_{j}\right)\right)^{2}+\left(\sup \gamma_{L}^{-}\left(x_{j}\right)-\sup \gamma_{M}^{-}\left(x_{j}\right)\right)^{2}\right]\end{array}\right\}$

It is clear that the bigger the value of $\operatorname{SM}_{\mathrm{j}}(L, M)(\mathrm{j}=1,2)$ represents $L$ is more similar to $M$.

Definition 6: Consider $L$ and $M$ be two IBNSs in $X=\left\{x_{1}, x_{2}, \ldots, x_{n}\right\}$. The similarity measure on the basis of minimum and maximum operators is defined as follows: 


$$
\left(\begin{array}{l}
\frac{\min \left(\inf \alpha_{L}^{+}\left(x_{i}\right), \inf \alpha_{M}^{+}\left(x_{i}\right)\right)}{\max \left(\inf \alpha_{L}^{+}\left(x_{i}\right), \inf \alpha_{M}^{+}\left(x_{i}\right)\right.}+\frac{\min \left(\sup \alpha_{L}^{+}\left(x_{i}\right), \sup \alpha_{M}^{+}\left(x_{i}\right)\right)}{\max \left(\sup \alpha_{L}^{+}\left(x_{i}\right), \sup \alpha_{M}^{+}\left(x_{i}\right)\right)}+\frac{\min \left(\inf \beta_{L}^{+}\left(x_{i}\right), \inf \beta_{M}^{+}\left(x_{i}\right)\right)}{\max \left(\inf \beta_{L}^{+}\left(x_{i}\right), \inf \beta_{M}^{+}\left(x_{i}\right)\right)} \\
\frac{\min \left(\sup \beta_{L}^{+}\left(x_{i}\right), \sup \beta_{M}^{+}\left(x_{i}\right)\right)}{\max \left(\sup \beta_{L}^{+}\left(x_{i}\right), \sup \beta_{M}^{+}\left(x_{i}\right)\right)}+\frac{\min \left(\inf \gamma_{L}^{+}\left(x_{i}\right), \inf \gamma_{M}^{+}\left(x_{i}\right)\right)}{\max \left(\inf \gamma_{L}^{+}\left(x_{i}\right), \inf \gamma_{M}^{+}\left(x_{i}\right)\right)}+\frac{\min \left(\sup \gamma_{L}^{+}\left(x_{i}\right), \sup \gamma_{M}^{+}\left(x_{i}\right)\right)}{\max \left(\sup \gamma_{L}^{+}\left(x_{i}\right), \sup \gamma_{M}^{+}\left(x_{i}\right)\right)} \\
\frac{\min \left(\left(\left(-\inf \alpha_{L}^{-}\left(x_{i}\right)\right),\left(-\inf \alpha_{M}^{-}\left(x_{i}\right)\right)\right)\right.}{\max \left(\left(\left(-\inf \alpha_{L}^{-}\left(x_{i}\right)\right),\left(-\inf \alpha_{M}^{-}\left(x_{i}\right)\right)\right)\right.}+\frac{\min \left(\left(\left(-\sup \alpha_{L}^{-}\left(x_{i}\right)\right),\left(-\sup \alpha_{M}^{-}\left(x_{i}\right)\right)\right)\right.}{\max \left(\left(\left(\sup \left(-\alpha_{L}^{-}\left(x_{i}\right)\right), \sup \left(-\alpha_{M}^{-}\left(x_{i}\right)\right)\right)\right.\right.}+ \\
\frac{\min \left(\left(\left(-\inf \beta_{L}^{-}\left(x_{i}\right)\right),\left(-\inf \beta_{M}^{-}\left(x_{i}\right)\right)\right)\right.}{\max \left(\left(\left(-\inf \beta_{L}^{-}\left(x_{i}\right)\right), \inf \left(-\beta_{M}^{-}\left(x_{i}\right)\right)\right)\right.}+\frac{\min \left(\left(\left(-\sup \beta_{L}^{-}\left(x_{i}\right)\right),\left(-\sup \beta_{M}^{-}\left(x_{i}\right)\right)\right)\right.}{\max \left(\left(\left(-\sup \beta_{L}^{-}\left(x_{i}\right)\right),\left(-\sup \beta_{M}^{-}\left(x_{i}\right)\right)\right)\right.}+ \\
\frac{\min \left(\left(\left(-\inf \gamma_{L}^{-}\left(x_{i}\right)\right),\left(-\inf \gamma_{M}^{-}\left(x_{i}\right)\right)\right)\right.}{\max \left(\left(\left(-\inf \gamma_{L}^{-}\left(x_{i}\right)\right),\left(-\inf \gamma_{M}^{-}\left(x_{i}\right)\right)\right)\right.}+\frac{\min \left(\left(\left(-\sup \gamma_{L}^{-}\left(x_{i}\right)\right),\left(-\sup \gamma_{M}^{-}\left(x_{i}\right)\right)\right)\right.}{\max \left(\left(\left(-\sup \gamma_{L}^{-}\left(x_{i}\right)\right),\left(-\sup \gamma_{M}^{-}\left(x_{i}\right)\right)\right)\right.}
\end{array}\right)
$$

Theorem 2. The interval bipolar neutrosophic similarity measure $Q_{1}(L, M)$ should satisfy the following properties:

(Q1) $0 \leq Q_{1}(L, M) \leq 1$;

(Q2) $Q_{1}(L, M)=1$, if $L=M$;

(Q3) $Q_{1}(L, M)=Q_{1}(M, L)$;

(Q4) If $L \subseteq M \subseteq N$, then $Q_{1}(L, N) \leq Q_{1}(L, M)$ and $Q_{1}(L, N) \leq Q_{1}(M, N)$ for an IBNS $N$ in $X$. Proof

$\left(Q_{1}\right) \quad$ From the definition 9, we have $0 \leq Q_{1}(L, M) \leq 1$.

$\left(\mathrm{Q}_{2}\right)$ If $\inf \alpha_{L}^{+}(x)=\inf \alpha_{M}^{+}(x), \sup \alpha_{L}^{+}(x)=\sup \alpha_{M}^{+}(x), \inf \beta_{L}^{+}(x)=\inf \beta_{M}^{+}(x), \sup \beta_{L}^{+}(x)=\sup \beta_{M}^{+}(x)$, $\inf \gamma_{L}^{+}(x)=\inf \gamma_{M}^{+}(x), \sup \gamma_{L}^{+}(x)=\sup \gamma_{M}^{+}(x), \inf \alpha_{L}^{-}(x)=\inf \alpha_{M}^{-}(x), \sup \alpha_{L}^{-}(x)=\sup \alpha_{M}^{-}(x), \inf \beta_{L}^{-}(x)=$ $\inf \beta_{M}^{-}(x), \sup \beta_{L}^{-}(x)=\sup \beta_{M}^{-}(x), \inf \gamma_{L}^{-}(x)=\inf \gamma_{M}^{-}(x), \sup \gamma_{L}^{-}(x)=\sup \gamma_{M}^{-}(x)$, for all $x \in X$, then obviously, $Q_{1}(L, M)=1$.

(Q3) $Q_{1}(L, M)=\frac{1}{12 n} \sum_{i=1}^{n}$

$\left(\begin{array}{l}\frac{\min \left(\inf \alpha_{L}^{+}\left(x_{i}\right), \inf \alpha_{M}^{+}\left(x_{i}\right)\right)}{\max \left(\inf \alpha_{L}^{+}\left(x_{i}\right), \inf \alpha_{M}^{+}\left(x_{i}\right)\right.}+\frac{\min \left(\sup \alpha_{L}^{+}\left(x_{i}\right), \sup \alpha_{M}^{+}\left(x_{i}\right)\right)}{\max \left(\sup \alpha_{L}^{+}\left(x_{i}\right), \sup \alpha_{M}^{+}\left(x_{i}\right)\right)}+\frac{\min \left(\inf \beta_{L}^{+}\left(x_{i}\right), \inf \beta_{M}^{+}\left(x_{i}\right)\right)}{\max \left(\inf \beta_{L}^{+}\left(x_{i}\right), \inf \beta_{M}^{+}\left(x_{i}\right)\right)}+ \\ \frac{\min \left(\sup \beta_{L}^{+}\left(x_{i}\right), \sup \beta_{M}^{+}\left(x_{i}\right)\right)}{\max \left(\sup \beta_{L}^{+}\left(x_{i}\right), \sup \beta_{M}^{+}\left(x_{i}\right)\right)}+\frac{\min \left(\inf \gamma_{L}^{+}\left(x_{i}\right), \inf \gamma_{M}^{+}\left(x_{i}\right)\right)}{\max \left(\inf \gamma_{L}^{+}\left(x_{i}\right), \inf \gamma_{M}^{+}\left(x_{i}\right)\right)}+\frac{\min \left(\sup \gamma_{L}^{+}\left(x_{i}\right), \sup \gamma_{M}^{+}\left(x_{i}\right)\right)}{\max \left(\sup \gamma_{L}^{+}\left(x_{i}\right), \sup \gamma_{M}^{+}\left(x_{i}\right)\right)}+ \\ \frac{\min \left(\left(\left(-\inf \alpha_{L}^{-}\left(x_{i}\right)\right),\left(-\inf \alpha_{M}^{-}\left(x_{i}\right)\right)\right)\right.}{\max \left(\left(\left(-\left(\inf \alpha_{L}^{-}\left(x_{i}\right)\right),\left(-\inf \alpha_{M}^{-}\left(x_{i}\right)\right)\right)\right.\right.}+\frac{\min \left(\left(\left(-\sup \alpha_{L}^{-}\left(x_{i}\right)\right),\left(-\sup \alpha_{M}^{-}\left(x_{i}\right)\right)\right)\right.}{\max \left(\left(\left(\sup \left(-\alpha_{L}^{-}\left(x_{i}\right)\right), \sup \left(-\alpha_{M}^{-}\left(x_{i}\right)\right)\right)\right.\right.}+ \\ \frac{\min \left(\left(\left(-\inf \beta_{L}^{-}\left(x_{i}\right)\right),\left(-\inf \beta_{M}^{-}\left(x_{i}\right)\right)\right)\right.}{\max \left(\left(\left(-\inf \beta_{L}^{-}\left(x_{i}\right)\right), \inf \left(-\beta_{M}^{-}\left(x_{i}\right)\right)\right)\right.}+\frac{\min \left(\left(\left(-\sup \beta_{L}^{-}\left(x_{i}\right)\right),\left(-\sup \beta_{M}^{-}\left(x_{i}\right)\right)\right)\right.}{\max \left(\left(\left(-\sup \beta_{L}^{-}\left(x_{i}\right)\right),\left(-\sup \beta_{M}^{-}\left(x_{i}\right)\right)\right)\right.}+ \\ \frac{\min \left(\left(\left(-\inf \gamma_{L}^{-}\left(x_{i}\right)\right),\left(-\inf \gamma_{M}^{-}\left(x_{i}\right)\right)\right)\right.}{\max \left(\left(\left(-\inf \gamma_{L}^{-}\left(x_{i}\right)\right),\left(-\inf \gamma_{M}^{-}\left(x_{i}\right)\right)\right)\right.}+\frac{\min \left(\left(\left(-\sup \gamma_{L}^{-}\left(x_{i}\right)\right),\left(-\sup \gamma_{M}^{-}\left(x_{i}\right)\right)\right)\right.}{\max \left(\left(\left(-\sup \gamma_{L}^{-}\left(x_{i}\right)\right),\left(-\sup \gamma_{M}^{-}\left(x_{i}\right)\right)\right)\right.}\end{array}\right)$

$$
=\frac{1}{12 n} \sum_{i=1}^{n}\left(\begin{array}{l}
\frac{\min \left(\inf \alpha_{M}^{+}\left(x_{i}\right), \inf \alpha_{L}^{+}\left(x_{i}\right)\right)}{\max \left(\inf \alpha_{M}^{+}\left(x_{i}\right), \inf \alpha_{L}^{+}\left(x_{i}\right)\right.}+\frac{\min \left(\sup \alpha_{M}^{+}\left(x_{i}\right), \sup \alpha_{L}^{+}\left(x_{i}\right)\right)}{\max \left(\sup \alpha_{M}^{+}\left(x_{i}\right), \sup \alpha_{L}^{+}\left(x_{i}\right)\right)}+\frac{\min \left(\inf \beta_{M}^{+}\left(x_{i}\right), \inf \beta_{L}^{+}\left(x_{i}\right)\right)}{\max \left(\inf \beta_{M}^{+}\left(x_{i}\right), \inf \beta_{L}^{+}\left(x_{i}\right)\right)}+ \\
\frac{\min \left(\sup \beta_{M}^{+}\left(x_{i}\right), \sup \beta_{L}^{+}\left(x_{i}\right)\right)}{\max \left(\sup \beta_{M}^{+}\left(x_{i}\right), \sup \beta_{L}^{+}\left(x_{i}\right)\right)}+\frac{\min \left(\inf \gamma_{M}^{+}\left(x_{i}\right), \inf \gamma_{L}^{+}\left(x_{i}\right)\right)}{\max \left(\inf \gamma_{M}^{+}\left(x_{i}\right), \inf \gamma_{L}^{+}\left(x_{i}\right)\right)}+\frac{\min \left(\sup \gamma_{M}^{+}\left(x_{i}\right), \sup \gamma_{L}^{+}\left(x_{i}\right)\right)}{\max \left(\sup \gamma_{M}^{+}\left(x_{i}\right), \sup \gamma_{L}^{+}\left(x_{i}\right)\right)}+ \\
\frac{\min \left(\left(\left(-\inf \alpha_{M}^{-}\left(x_{i}\right)\right),\left(-\inf \alpha_{L}^{-}\left(x_{i}\right)\right)\right)\right.}{\max \left(\left(\left(-\left(\inf \alpha_{M}^{-}\left(x_{i}\right)\right),\left(-\inf \alpha_{L}^{-}\left(x_{i}\right)\right)\right)\right.\right.}+\frac{\min \left(\left(\left(-\sup \alpha_{M}^{-}\left(x_{i}\right)\right),\left(-\sup \alpha_{L}^{-}\left(x_{i}\right)\right)\right)\right.}{\max \left(\left(\left(\sup \left(-\alpha_{M}^{-}\left(x_{i}\right)\right), \sup \left(-\alpha_{L}^{-}\left(x_{i}\right)\right)\right)\right.\right.}+ \\
\frac{\min \left(\left(\left(-\inf \beta_{M}^{-}\left(x_{i}\right)\right),\left(-\inf \beta_{L}^{-}\left(x_{i}\right)\right)\right)\right.}{\max \left(\left(\left(-\inf \beta_{M}^{-}\left(x_{i}\right)\right), \inf \left(-\beta_{L}^{-}\left(x_{i}\right)\right)\right)\right.}+\frac{\min \left(\left(\left(-\sup \beta_{M}^{-}\left(x_{i}\right)\right),\left(-\sup \beta_{L}^{-}\left(x_{i}\right)\right)\right)\right.}{\max \left(\left(\left(-\sup \beta_{M}^{-}\left(x_{i}\right)\right),\left(-\sup \beta_{L}^{-}\left(x_{i}\right)\right)\right)\right.}+ \\
\frac{\min \left(\left(\left(-\inf \gamma_{M}^{-}\left(x_{i}\right)\right),\left(-\inf \gamma_{L}^{-}\left(x_{i}\right)\right)\right)\right.}{\max \left(\left(\left(-\inf \gamma_{M}^{-}\left(x_{i}\right)\right),\left(-\inf \gamma_{L}^{-}\left(x_{i}\right)\right)\right)\right.}+\frac{\min \left(\left(\left(-\sup \gamma_{M}^{-}\left(x_{i}\right)\right),\left(-\sup \gamma_{L}^{-}\left(x_{i}\right)\right)\right)\right.}{\max \left(\left(\left(-\sup \gamma_{M}^{-}\left(x_{i}\right)\right),\left(-\sup \gamma_{L}^{-}\left(x_{i}\right)\right)\right)\right.}
\end{array}\right)=Q_{1}(M, L) .
$$


$\left(Q_{4}\right)$ Let, $L \subseteq M \subseteq N$, then $\inf \alpha_{L}^{+}(x) \leq \inf \alpha_{M}^{+}(x) \leq \inf \alpha_{N}^{+}(x), \sup \alpha_{L}^{+}(x) \leq \sup \alpha_{M}^{+}(x) \leq \sup \alpha_{N}^{+}(x)$, inf $\beta_{L}^{+}(x) \geq \inf \beta_{M}^{+}(x) \geq \inf \beta_{N}^{+}(x), \sup \beta_{L}^{+}(x) \geq \sup \beta_{M}^{+}(x) \geq \sup \beta_{N}^{+}(x), \inf \gamma_{L}^{+}(x) \geq \inf \gamma_{M}^{+}(x) \geq \inf \gamma_{N}^{+}(x)$, $\sup \gamma_{L}^{+}(x) \geq \sup \gamma_{M}^{+}(x) \geq \sup \gamma_{N}^{+}(x),-\inf \alpha_{L}^{-}(x) \leq-\inf \alpha_{M}^{-}(x) \leq-\inf \alpha_{N}^{-}(x), \quad-\sup \alpha_{L}^{-}(x) \leq-\sup \alpha_{M}(x) \leq$ $-\sup \alpha_{N}^{-}(x), \quad-\inf \beta_{L}^{-}(x) \geq-\inf \beta_{M}^{-}(x) \geq-\inf \beta_{N}^{-}(x), \quad-\sup \beta_{L}^{-}(x) \geq-\sup \beta_{M}^{-}(x) \geq-\sup \beta_{N}^{-}(x), \quad-\inf \gamma_{L}^{-}(x) \geq-\inf$ $\gamma_{M}^{-}(x) \geq-\inf \gamma_{N}^{-}(x),-\sup \gamma_{L}^{-}(x) \geq-\sup \gamma_{M}^{-}(x) \geq-\sup \gamma_{N}^{-}(x)$ for every $x \in X$. Using these inequalities, we have the following similarity measures:

$Q_{1}(L, M)=\frac{1}{12 n} \sum_{i=1}^{n}\left\{\begin{array}{l}\frac{\inf \alpha_{L}^{+}\left(x_{i}\right)}{\inf \alpha_{M}^{+}\left(x_{i}\right)}+\frac{\sup \alpha_{L}^{+}\left(x_{i}\right)}{\sup \alpha_{M}^{+}\left(x_{i}\right)}+\frac{\inf \beta_{M}^{+}\left(x_{i}\right)}{\inf \beta_{L}^{+}\left(x_{i}\right)}+\frac{\sup \beta_{M}^{+}\left(x_{i}\right)}{\sup \beta_{L}^{+}\left(x_{i}\right)}+\frac{\inf \gamma_{M}^{+}\left(x_{i}\right)}{\inf \gamma_{L}^{+}\left(x_{i}\right)}+\frac{\sup \gamma_{M}^{+}\left(x_{i}\right)}{\sup \gamma_{L}^{+}\left(x_{i}\right)}+ \\ \frac{\left(-\inf \alpha_{L}^{-}\left(x_{i}\right)\right)}{\left(-\inf \alpha_{M}^{-}\left(x_{i}\right)\right)}+\frac{\left(-\sup \alpha_{L}^{-}\left(x_{i}\right)\right)}{\left(-\sup \alpha_{M}^{-}\left(x_{i}\right)\right)}+\frac{\left(-\inf \beta_{M}^{-}\left(x_{i}\right)\right)}{\left(-\inf \beta_{L}^{-}\left(x_{i}\right)\right)}+\frac{\left(-\sup \beta_{M}^{-}\left(x_{i}\right)\right)}{\left(-\sup \beta_{L}^{-}\left(x_{i}\right)\right)}+\frac{\left(-\inf \gamma_{M}^{-}\left(x_{i}\right)\right)}{\left(-\inf \gamma_{L}^{-}\left(x_{i}\right)\right)}+\frac{\left(-\sup \gamma_{M}^{-}\left(x_{i}\right)\right)}{\left(-\sup \gamma_{L}^{-}\left(x_{i}\right)\right)}\end{array}\right\rangle$

$Q_{1}(L, N)=\frac{1}{12 n} \sum_{i=1}^{n}\left\{\begin{array}{l}\frac{\inf \alpha_{L}^{+}\left(x_{i}\right)}{\inf \alpha_{N}^{+}\left(x_{i}\right)}+\frac{\sup \alpha_{L}^{+}\left(x_{i}\right)}{\sup \alpha_{N}^{+}\left(x_{i}\right)}+\frac{\inf \beta_{N}^{+}\left(x_{i}\right)}{\inf \beta_{L}^{+}\left(x_{i}\right)}+\frac{\sup \beta_{N}^{+}\left(x_{i}\right)}{\sup I_{L}^{+}\left(x_{i}\right)}+\frac{\inf F_{N}^{+}\left(x_{i}\right)}{\inf F_{L}^{+}\left(x_{i}\right)}+\frac{\sup F_{N}^{+}\left(x_{i}\right)}{\sup F_{L}^{+}\left(x_{i}\right)}+ \\ \frac{\left(-\inf \alpha_{L}^{-}\left(x_{i}\right)\right)}{\left(-\inf \alpha_{N}^{-}\left(x_{i}\right)\right)}+\frac{\left(-\sup \alpha_{L}^{-}\left(x_{i}\right)\right)}{\left(-\sup \alpha_{N}^{-}\left(x_{i}\right)\right)}+\frac{\left(-\inf \beta_{N}^{-}\left(x_{i}\right)\right)}{\left(-\inf \beta_{L}^{-}\left(x_{i}\right)\right)}+\frac{\left(-\sup \beta_{N}^{-}\left(x_{i}\right)\right)}{\left(-\sup \beta_{L}^{-}\left(x_{i}\right)\right)}+\frac{\left(-\inf \gamma_{N}^{-}\left(x_{i}\right)\right)}{\left(-\inf \gamma_{L}^{-}\left(x_{i}\right)\right)}+\frac{\left(-\sup \gamma_{N}^{-}\left(x_{i}\right)\right)}{\left(-\sup \gamma_{L}^{-}\left(x_{i}\right)\right)}\end{array}\right\rangle$

$Q_{1}(M, N)=\frac{1}{12 n} \sum_{i=1}^{n}\left(\begin{array}{l}\frac{\inf \alpha_{M}^{+}\left(x_{i}\right)}{\inf \alpha_{N}^{+}\left(x_{i}\right)}+\frac{\sup \alpha_{M}^{+}\left(x_{i}\right)}{\sup \alpha_{N}^{+}\left(x_{i}\right)}+\frac{\inf \beta_{N}^{+}\left(x_{i}\right)}{\inf \beta_{M}^{+}\left(x_{i}\right)}+\frac{\sup \beta_{N}^{+}\left(x_{i}\right)}{\sup \beta_{M}^{+}\left(x_{i}\right)}+\frac{\inf \gamma_{N}^{+}\left(x_{i}\right)}{\inf \gamma_{M}^{+}\left(x_{i}\right)}+\frac{\sup \gamma_{N}^{+}\left(x_{i}\right)}{\sup \gamma_{M}^{+}\left(x_{i}\right)}+ \\ \frac{\left(-\inf \alpha_{M}^{-}\left(x_{i}\right)\right)}{\left(-\inf \alpha_{N}^{-}\left(x_{i}\right)\right)}+\frac{\left(-\sup \alpha_{M}^{-}\left(x_{i}\right)\right)}{\left(-\sup \alpha_{N}^{-}\left(x_{i}\right)\right)}+\frac{\left(-\inf \beta_{N}^{-}\left(x_{i}\right)\right)}{\left(-\inf \beta_{M}^{-}\left(x_{i}\right)\right)}+\frac{\left(-\sup \beta_{N}^{-}\left(x_{i}\right)\right)}{\left(-\sup \beta_{M}^{-}\left(x_{i}\right)\right)}+\frac{\left(-\inf \gamma_{N}^{-}\left(x_{i}\right)\right)}{\left(-\inf \gamma_{M}^{-}\left(x_{i}\right)\right)}+\frac{\left(-\sup \gamma_{N}^{-}\left(x_{i}\right)\right)}{\left(-\sup \gamma_{M}^{-}\left(x_{i}\right)\right)}\end{array}\right)$

Since, we have

$\frac{\inf \alpha_{L}^{+}\left(x_{i}\right)}{\inf \alpha_{N}^{+}\left(x_{i}\right)} \leq \frac{\inf \alpha_{L}^{+}\left(x_{i}\right)}{\inf \alpha_{M}^{+}\left(x_{i}\right)} ; \frac{\sup \alpha_{L}^{+}\left(x_{i}\right)}{\sup \alpha_{N}^{+}\left(x_{i}\right)} \leq \frac{\sup \alpha_{L}^{+}\left(x_{i}\right)}{\sup \alpha_{M}^{+}\left(x_{i}\right)} ; \frac{\inf \beta_{N}^{+}\left(x_{i}\right)}{\inf \beta_{L}^{+}\left(x_{i}\right)} \leq \frac{\inf \beta_{M}^{+}\left(x_{i}\right)}{\inf \beta_{L}^{+}\left(x_{i}\right)} ; \frac{\sup \beta_{N}^{+}\left(x_{i}\right)}{\sup \beta_{L}^{+}\left(x_{i}\right)} \leq$ $\frac{\sup \beta_{M}^{+}\left(x_{i}\right)}{\sup \beta_{L}^{+}\left(x_{i}\right)} \quad ; \quad \frac{\inf \gamma_{N}^{+}\left(x_{i}\right)}{\inf \gamma_{L}^{+}\left(x_{i}\right)} \leq \frac{\inf \gamma_{M}^{+}\left(x_{i}\right)}{\inf \gamma_{L}^{+}\left(x_{i}\right)} \quad ; \quad \frac{\sup \gamma_{N}^{+}\left(x_{i}\right)}{\sup \gamma_{L}^{+}\left(x_{i}\right)} \leq \frac{\sup \gamma_{M}^{+}\left(x_{i}\right)}{\sup \gamma_{L}^{+}\left(x_{i}\right)} ;$ $\frac{\left(-\inf \alpha_{L}^{-}\left(x_{i}\right)\right)}{\left(-\inf \alpha_{N}^{-}\left(x_{i}\right)\right)} \leq \frac{\left(-\inf \alpha_{L}^{-}\left(x_{i}\right)\right)}{\left(-\inf \alpha_{M}^{-}\left(x_{i}\right)\right)} ; \frac{\left(-\sup \alpha_{L}^{-}\left(x_{i}\right)\right)}{\left(-\sup \alpha_{N}^{-}\left(x_{i}\right)\right)} \leq \frac{\left(-\sup \alpha_{L}^{-}\left(x_{i}\right)\right)}{\left(-\sup \alpha_{M}^{-}\left(x_{i}\right)\right)} ; \frac{\left(-\inf \beta_{N}^{-}\left(x_{i}\right)\right)}{\left(-\inf \beta_{L}^{-}\left(x_{i}\right)\right)} \leq \frac{\left(-\inf \beta_{M}^{-}\left(x_{i}\right)\right)}{\left(-\inf \beta_{L}^{-}\left(x_{i}\right)\right)} ;$ $\frac{\left(-\sup \beta_{N}^{-}\left(x_{i}\right)\right)}{\left(-\sup \beta_{L}^{-}\left(x_{i}\right)\right)} \leq \frac{\left(-\sup \beta_{M}^{-}\left(x_{i}\right)\right)}{\left(-\sup \beta_{L}^{-}\left(x_{i}\right)\right)} ; \frac{\left(-\inf \gamma_{N}^{-}\left(x_{i}\right)\right)}{\left(-\inf \gamma_{L}^{-}\left(x_{i}\right)\right)} \leq \frac{\left(-\inf \gamma_{M}^{-}\left(x_{i}\right)\right)}{\left(-\inf \gamma_{L}^{-}\left(x_{i}\right)\right)} ; \frac{\left(-\sup \gamma_{N}^{-}\left(x_{i}\right)\right)}{\left(-\sup \gamma_{L}^{-}\left(x_{i}\right)\right)} \leq \frac{\left(-\sup \gamma_{M}^{-}\left(x_{i}\right)\right)}{\left(-\sup \gamma_{L}^{-}\left(x_{i}\right)\right)}$ we can obtain that $Q_{1}(L, N) \leq Q_{1}(L, M)$.

Similarly, we have $\frac{\inf \alpha_{L}^{+}\left(x_{i}\right)}{\inf \alpha_{N}^{+}\left(x_{i}\right)} \leq \frac{\inf \alpha_{M}^{+}\left(x_{i}\right)}{\inf \alpha_{N}^{+}\left(x_{i}\right)} ; \frac{\sup \alpha_{L}^{+}\left(x_{i}\right)}{\sup \alpha_{N}^{+}\left(x_{i}\right)} \leq \frac{\sup \alpha_{M}^{+}\left(x_{i}\right)}{\sup \alpha_{N}^{+}\left(x_{i}\right)} ; \frac{\inf \beta_{N}^{+}\left(x_{i}\right)}{\inf \beta_{L}^{+}\left(x_{i}\right)} \leq \frac{\inf \beta_{N}^{+}\left(x_{i}\right)}{\inf \beta_{M}^{+}\left(x_{i}\right)} ; \frac{\sup \beta_{N}^{+}\left(x_{i}\right)}{\sup \beta_{L}^{+}\left(x_{i}\right)} \leq$ $\frac{\sup \beta_{N}^{+}\left(x_{i}\right)}{\sup \beta_{M}^{+}\left(x_{i}\right)} ; \frac{\inf \gamma_{N}^{+}\left(x_{i}\right)}{\inf \gamma_{L}^{+}\left(x_{i}\right)} \leq \frac{\inf \gamma_{N}^{+}\left(x_{i}\right)}{\inf \gamma_{M}^{+}\left(x_{i}\right)} ; \frac{\sup \gamma_{N}^{+}\left(x_{i}\right)}{\sup \gamma_{L}^{+}\left(x_{i}\right)} \leq \frac{\sup \gamma_{N}^{+}\left(x_{i}\right)}{\sup \gamma_{M}^{+}\left(x_{i}\right)} ; \frac{\left(-\inf \alpha_{L}^{-}\left(x_{i}\right)\right)}{\left(-\inf \alpha_{N}^{-}\left(x_{i}\right)\right)} \leq \frac{\left(-\inf \alpha_{M}^{-}\left(x_{i}\right)\right.}{\left(-\inf \alpha_{N}^{-}\left(x_{i}\right)\right)} ;$ 
10 of 18

$$
\begin{aligned}
& \frac{\left(-\sup \alpha_{L}^{-}\left(x_{i}\right)\right)}{\left(-\sup \alpha_{N}^{-}\left(x_{i}\right)\right)} \leq \frac{\left(-\sup \alpha_{M}^{-}\left(x_{i}\right)\right)}{\left(-\sup \alpha_{N}^{-}\left(x_{i}\right)\right)} ; \frac{\left(-\inf \beta_{N}^{-}\left(x_{i}\right)\right)}{\left(-\inf \beta_{L}^{-}\left(x_{i}\right)\right)} \leq \frac{\left(-\inf \beta_{N}^{-}\left(x_{i}\right)\right)}{\left(-\inf \beta_{M}^{-}\left(x_{i}\right)\right)} ; \frac{\left(-\sup \beta_{N}^{-}\left(x_{i}\right)\right)}{\left(-\sup \beta_{L}^{-}\left(x_{i}\right)\right)} \leq \frac{\left(-\sup \beta_{N}^{-}\left(x_{i}\right)\right)}{\left(-\sup \beta_{M}^{-}\left(x_{i}\right)\right)} \\
& \frac{\left(-\inf \gamma_{N}^{-}\left(x_{i}\right)\right)}{\left(-\inf \gamma_{L}^{-}\left(x_{i}\right)\right)} \leq \frac{\left(-\inf \gamma_{N}^{-}\left(x_{i}\right)\right)}{\left(-\inf \gamma_{M}^{-}\left(x_{i}\right)\right)} ; \frac{\left(-\sup \gamma_{N}^{-}\left(x_{i}\right)\right)}{\left(-\sup \gamma_{L}^{-}\left(x_{i}\right)\right)} \leq \frac{\left(-\sup \gamma_{N}^{-}\left(x_{i}\right)\right)}{\left(-\sup \gamma_{M}^{-}\left(x_{i}\right)\right)}
\end{aligned}
$$

Therefore, we obtain $Q_{1}(L, N) \leq Q_{1}(M, N)$.

Example 2. Suppose that $L=<[0.2,0.8],[0.1,0.2],[0.2,0.7],[-0.4,-0.3],[-0.5,-0.3],[-0.8,-0.5]>$ and $M=<[0.4,0.6],[0.5,0.8],[0.3,0.9],[-0.8,-0.1],[-0.8,-0.6],[-0.6,-0.4]>$ be two IBNSs, then the similarity measure $Q_{1}(L, M)$ between $L$ and $M$ is given by

$$
Q_{1}(L, M)=\frac{1}{12} \times\left(\begin{array}{l}
\frac{0.2}{0.4}+\frac{0.6}{0.8}+\frac{0.1}{0.5}+\frac{0.1}{0.5}+\frac{0.2}{0.8}+\frac{0.7}{0.9}+\frac{-(-0.4)}{-(-0.8)}+\frac{-(-0.1)}{-(-0.3)}+ \\
\frac{-(-0.5)}{-(-0.8)}+\frac{-(-0.3)}{-(-0.6)}+\frac{-(-0.6)}{-(-0.8)}+\frac{-(-0.4)}{-(-0.5)}
\end{array}\right\rangle=0.5155
$$

However, if we consider the importance differences in the independent components (i.e., positive and negative truth-membership $\alpha^{+}, \alpha^{-}$, indeterminacy-membership $\beta^{+}, \beta^{-}$and falsity-membership $\gamma^{+}, \gamma^{-}$where $\left.\alpha^{+}, \beta^{+}, \gamma^{+} \subseteq[0,1] ; \alpha^{-}, \beta^{-}, \gamma^{-} \subseteq[-1,0]\right)$ in a IBNS, we can input the weights of the independent components in Eq. (9). Therefore, we define another similarity measure between $L$ and $M$ as follows:

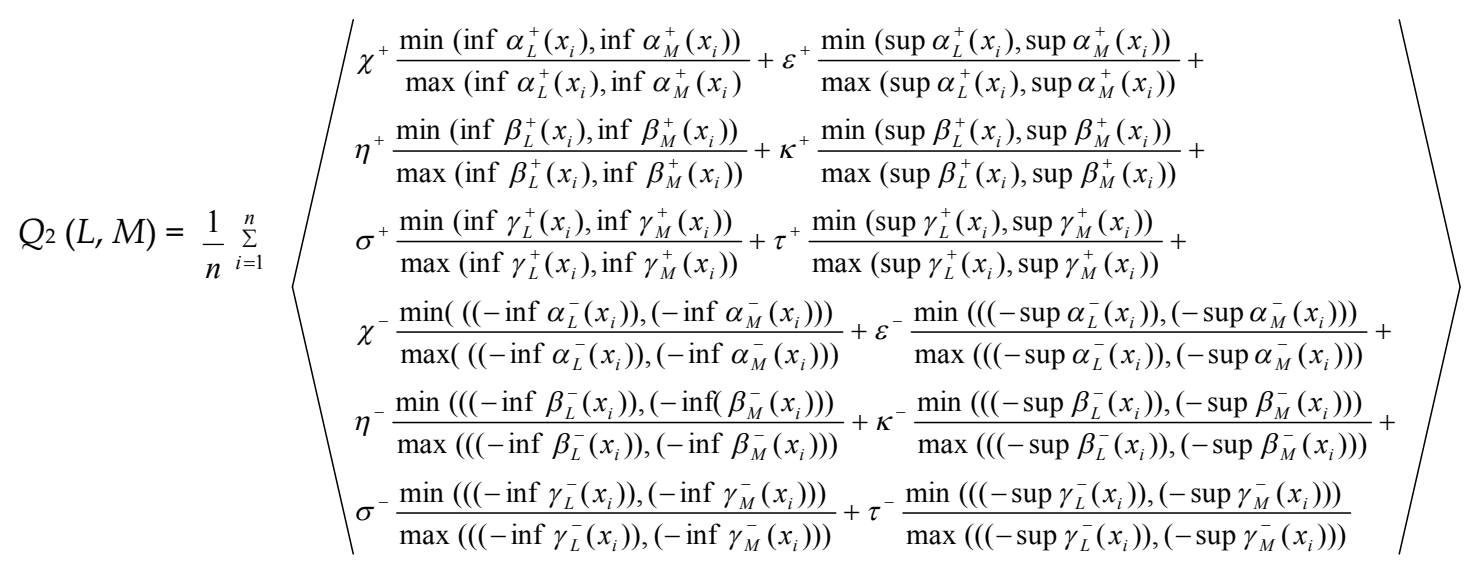

where $\chi^{+}, \varepsilon^{+}, \eta^{+}, \kappa^{+}, \sigma^{+}, \tau^{+}, \chi^{-}, \varepsilon^{-}, \eta^{-}, \kappa^{-}, \sigma^{-}, \tau^{-}$are the weights of the independent components in a IBNS and $\chi^{+}+\varepsilon^{+}+\eta^{+}+\kappa^{+}+\sigma^{+}+\tau^{+}+\chi^{-}+\varepsilon^{-}+\eta^{-}+\kappa^{-}+\sigma^{-}+\tau^{-}=1$. Especially, when $\chi^{+}=\varepsilon^{+}=\eta^{+}=\kappa^{+}=\sigma^{+}=\tau^{+}=\chi^{-}=\varepsilon^{-}=\eta^{-}=\kappa^{-}=\sigma^{-}=\tau^{-}=1 / 12$, Eq (10) reduces to Eq. (9).

Furthermore, if we consider the importance dissimilarities in $X=\left\{x_{1}, x_{2}, \ldots, x_{n}\right\}$, we require to consider the weight of every point $x_{\mathrm{i}}(\mathrm{i}=1,2, \ldots, n)$. Therefore, we construct another similarity measure between $L$ and $M$ as given below. 


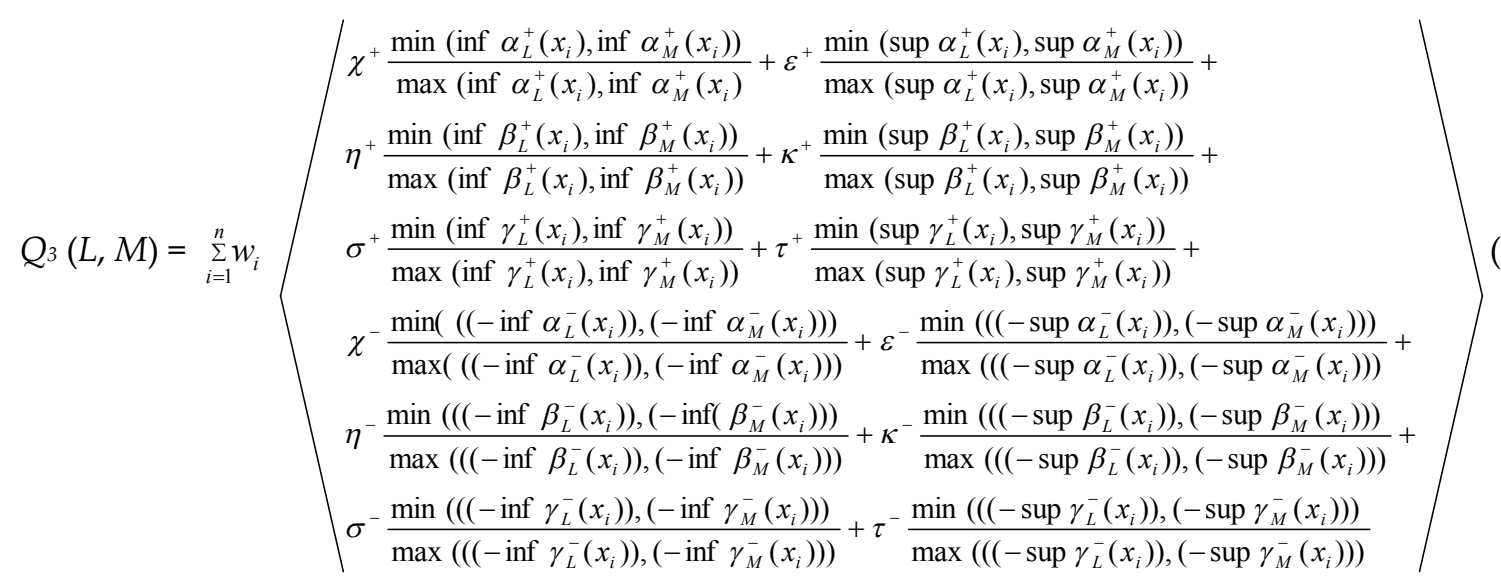

Here, $w_{\mathrm{i}}$ be the weight of each object $x_{\mathrm{i}}(\mathrm{i}=1,2, \ldots, n)$ such that $0 \leq w_{\mathrm{i}} \leq 1$ and $\sum_{\mathrm{i}=1}^{n} w_{\mathrm{i}}=1$. If $w=(1 / n$, $1 / n, \ldots, 1 / n)^{\mathrm{T}}$, then Eq. (11) reduces to Eq. (10).

Theorem 3. The interval bipolar neutrosophic similarity measure $Q_{j}(L, M), j=2,3$ should also satisfy the following properties:

(Q1) $0 \leq Q_{j}(L, M) \leq 1 ; j=2,3$
(Q2) $Q_{j}(L, M)=Q_{j}(M, L) ; j=2,33$
(Q3) $Q_{j}(L, M)=1$, if $L=M ; j=2,3$

(Q4) If $L \subseteq M \subseteq N$, then $Q_{\mathrm{j}}(L, N) \leq Q_{\mathrm{j}}(L, M)$ and $Q_{\mathrm{j}}(L, N) \leq Q_{\mathrm{j}}(L, M), \mathrm{j}=2,3$ for $L, M, N$ in X.

\section{Proof}

The properties of the Theorem 3 can be established in the same way as the Theorem 2.

Example 3 Suppose that $L=<\left\{x_{1},[0.2,0.8],[0.1,0.2],[0.2,0.7],[-0.4,-0.3],[-0.5,-0.3],[-0.8,-0.5]\right\}$, $\left\{x_{2},[0.4,0.9],[0.1,0.3],[0.6,0.9],[-0.5,-0.2],[-0.5,-0.1],[-0.8,-0.3]\right\}>$ and $M=<\left\{x_{1},[0.4,0.6],[0.5,0.8]\right.$, $[0.3,0.9],[-0.8,-0.1],[-0.8,-0.6],[-0.6,-0.4]\},\left\{x_{2},[0.1,0.7],[0.2,0.4],[0.2,0.6],[-0.4,-0.1],[-0.7,-0.3]\right.$, $[-0.6,-0.2]\}>$ be two IBNSs in $X=\left\{x_{1}, x_{2}\right\}$.

If the weight values of the independent elements in a IBNS are $\chi^{+}=0.05, \varepsilon^{+}=0.05, \eta^{+}=0.07$, $\kappa^{+}=0.07, \sigma^{+}=0.1, \tau^{+}=0.1, \chi^{-}=0.1, \varepsilon^{-}=0.1, \eta^{-}=0.07, \kappa^{-}=0.07, \sigma^{-}=0.11, \tau^{-}=0.11$, then the similarity measure $Q_{2}(L, M)$ between $L$ and $M$ is given by

$$
Q_{2}(L, M)=\frac{1}{2} \times\left(\begin{array}{l}
(0.05) \frac{0.2}{0.4}+(0.05) \frac{0.6}{0.8}+(0.07) \frac{0.1}{0.5}+(0.07) \frac{0.2}{0.8}+(0.1) \frac{0.2}{0.3}+(0.1) \frac{0.7}{0.9}+(0.1) \frac{-(-0.4)}{-(-0.8)}+(0.1) \frac{-(-0.1)}{-(-0.3)}+(0.07) \frac{-(-0.5)}{-(-0.8)}+ \\
(0.07) \frac{-(-0.3)}{-(-0.6)}+(0.11) \frac{-(-0.6)}{-(-0.8)}+(0.11) \frac{-(-0.4)}{-(-0.5)}+(0.05) \frac{0.1}{0.4}+(0.05) \frac{0.7}{0.9}+(0.07) \frac{0.1}{0.2}+(0.07) \frac{0.3}{0.4}+(0.1) \frac{0.2}{0.6}+ \\
(0.1) \frac{0.6}{0.9}+(0.1) \frac{-(-0.4)}{-(-0.5)}+(0.1) \frac{-(-0.1)}{-(-0.2)}+(0.07) \frac{-(-0.5)}{-(-0.7)}+(0.07) \frac{-(-0.1)}{-(-0.3)}+(0.11) \frac{-(-0.6)}{-(-0.8)}+(0.11) \frac{-(-0.2)}{-(-0.3)}
\end{array}\right\}
$$

$=0.5845$.

Assume that the weight vector of the attributes is $w=(0.3,0.7)^{\mathrm{T}}$ and the weight values of the independent elements in a IBNS are $\chi^{+}=0.05, \varepsilon^{+}=0.05, \eta^{+}=0.07, \kappa^{+}=0.07, \sigma^{+}=0.1, \tau^{+}=0.1$, 
$\chi^{-}=0.1, \varepsilon^{-}=0.1, \eta^{-}=0.07, \kappa^{-}=0.07, \sigma^{-}=0.11, \tau^{-}=0.11$, then the similarity measure $Q_{3}(L, M)$ between $L$ and $M$ is computed as follows.

$$
Q_{3}(L, M)=\left\{\begin{array}{l}
0.3 \times\left[(0.05) \frac{0.2}{0.4}+(0.05) \frac{0.6}{0.8}+(0.07) \frac{0.1}{0.5}+(0.07) \frac{0.2}{0.8}+(0.1) \frac{0.2}{0.3}+(0.1) \frac{0.7}{0.9}+(0.1) \frac{-(-0.4)}{-(-0.8)}+(0.1) \frac{-(-0.1)}{-(-0.3)}+(0.07) \frac{-(-0.5)}{-(-0.8)}+\right. \\
\left.(0.07) \frac{-(-0.3)}{-(-0.6)}+(0.11) \frac{-(-0.6)}{-(-0.8)}+(0.11) \frac{-(-0.4)}{-(-0.5)}\right]+0.7 \times\left[(0.05) \frac{0.1}{0.4}+(0.05) \frac{0.7}{0.9}+(0.07) \frac{0.1}{0.2}+(0.07) \frac{0.3}{0.4}+(0.1) \frac{0.2}{0.6}+\right. \\
\left.(0.1) \frac{0.6}{0.9}+(0.1) \frac{-(-0.4)}{-(-0.5)}+(0.1) \frac{-(-0.1)}{-(-0.2)}+(0.07) \frac{-(-0.5)}{-(-0.7)}+(0.07) \frac{-(-0.1)}{-(-0.3)}+(0.11) \frac{-(-0.6)}{-(-0.8)}+(0.11) \frac{-(-0.2)}{-(-0.3)}\right]
\end{array}\right\rangle
$$

$=0.5899$.

\section{Interval bipolar multi-attribute decision making strategies based on the proposed similarity measures}

In this section, we present three novel similarity measures for MADM problem in interval bipolar neutrosophic environment. Consider $Y=\left\{Y_{1}, Y_{2}, \ldots, Y_{m}\right\},(m \geq 2)$ be a discrete set of $m$ feasible alternatives, $Z=\left\{Z_{1}, Z_{2}, \ldots, Z_{n}\right\},(n \geq 2)$ be a set of attributes under consideration and $w_{\mathrm{j}}$ be the weight vector of the attributes such that $0 \leq w_{\mathrm{j}} \leq 1$ and $\sum_{\mathrm{j}=1}^{n} w_{\mathrm{j}}=1$. Three MADM strategies are presented in compact form as follows.

Step 1. The decision maker assigns the rating of performance value of alternative $Y_{i}(i=1,2, \ldots$, $m)$ with respect to the predefined attribute $Z_{j}(j=1,2, \ldots, n)$ in terms of interval bipolar neutrosophic values $p_{\mathrm{ij}}=<\left[\inf \alpha_{i j}^{+}, \sup \alpha_{i j}^{+}\right],\left[\inf \beta_{i j}^{+}, \sup \beta_{i j}^{+}\right],\left[\inf \gamma_{i j}^{+}, \sup \gamma_{i j}^{+}\right],\left[\inf \alpha_{i j}^{-}, \sup \alpha_{i j}^{-}\right],\left[\inf \beta_{i j}^{-}, \sup \beta_{i j}^{-}\right]$, $\left[\inf \gamma_{i j}^{-}, \sup \gamma_{i j}^{-}\right]>=\left\langle\left[a_{i j}, b_{i j}\right],\left[c_{i j}, d_{i j}\right],\left[e_{i j}, f_{i j}\right],\left[r_{i j}, s_{i j}\right],\left[t_{i j}, u_{i j}\right],\left[v_{i j}, w_{i j}\right]\right\rangle, \mathrm{i}=1,2, \ldots, m ; \mathrm{j}=1,2, \ldots, n$. The interval bipolar neutrosophic decision matrix $\left[\widetilde{D}_{i j}\right]_{m \times n}$ can be presented as follows:

$$
\left[\widetilde{D}_{i j}\right]_{m \times n}=\begin{array}{cccc}
Z_{1} & Z_{2} & \cdots & Z_{n} \\
Y_{1} & Y_{2} \\
\cdot & P_{m}
\end{array}\left(\begin{array}{cccc}
p_{11} & p_{12} & \cdots & p_{1 n} \\
p_{21} & p_{22} & \cdots & p_{2 n} \\
\cdot & \cdot & \cdots & \cdot \\
\cdot & \cdot & \cdots & \cdot \\
p_{m 1} & p_{m 2} & \cdots & p_{m n}
\end{array}\right)
$$

Step 2. The interval bipolar neutrosophic positive ideal solution (IBN-PIS) can be defined by utilizing a maximum operator for the benefit attribute and a minimum operator for the cost attribute as follows:

$$
\begin{aligned}
& Y^{+}=\left\langle\left[a_{\mathrm{j}}^{+}, b_{\mathrm{j}}^{+}\right],\left[c_{\mathrm{j}}^{+}, d_{\mathrm{j}}^{+}\right],\left[e_{\mathrm{j}}^{+}, f_{\mathrm{j}}^{+}\right],\left[r_{\mathrm{j}}^{-}, s_{\mathrm{j}}^{-}\right],\left[t_{\mathrm{j}}^{-}, u_{\mathrm{j}}^{-}\right],\left[v_{\mathrm{j}}^{-}, w_{\mathrm{j}}^{-}\right]\right\rangle=\left\langle\left[\left\{\underset{\mathrm{i}}{\operatorname{Max}}\left(a_{i j}\right)\left|\mathrm{j} \in H_{1} ; \underset{\mathrm{i}}{\operatorname{Min}}\left(a_{i j}\right)\right| \mathrm{j} \in H_{2}\right\},\right.\right. \\
& \left.\left.\left\{\operatorname{Max}_{\mathrm{i}}\left(b_{i j}\right) \mid \mathrm{j} \in H_{1}\right\} ; \operatorname{Min}_{\mathrm{i}}\left(b_{i j}\right) \mid \mathrm{j} \in H_{2}\right\}\right], \quad\left[\left\{\operatorname{Min}_{\mathrm{i}}\left(c_{i j}\right)\left|\mathrm{j} \in H_{1} ; \operatorname{Max}_{\mathrm{i}}\left(c_{i j}\right)\right| \mathrm{j} \in H_{2}\right\},\left\{\operatorname{Min}_{\mathrm{i}}\left(d_{i j}\right) \mid \mathrm{j} \in H_{1}\right\} ;\right. \\
& \left.\left.\left.\underset{\mathrm{i}}{\operatorname{Max}}\left(d_{i j}\right) \mid \mathrm{j} \in H_{2}\right\}\right], \quad\left[\left\{\operatorname{Min}_{\mathrm{i}}\left(e_{i j}\right)\left|\mathrm{j} \in H_{1} ; \operatorname{Max}_{\mathrm{i}}\left(e_{i j}\right)\right| \mathrm{j} \in H_{2}\right\}, \quad\left\{\operatorname{Min}_{\mathrm{i}}\left(f_{i j}\right) \mid \mathrm{j} \in H_{1}\right\} ; \quad \operatorname{Max}_{\mathrm{i}}\left(f_{i j}\right) \mid \mathrm{j} \in H_{2}\right\}\right], \\
& \left.\left[\left\{\operatorname{Min}_{\mathrm{i}}\left(r_{i j}\right)\left|\mathrm{j} \in H_{1} ; \operatorname{Max}_{\mathrm{i}}\left(r_{i j}\right)\right| \mathrm{j} \in H_{2}\right\},\left\{\operatorname{Min}_{\mathrm{i}}\left(s_{i j}\right) \mid \mathrm{j} \in H_{1}\right\} ; \quad \operatorname{Max}_{\mathrm{i}}\left(s_{i j}\right) \mid \mathrm{j} \in H_{2}\right\}\right], \quad\left[\left\{\operatorname{Max}\left(t_{i j}\right) \mid \mathrm{j} \in H_{1} ;\right.\right.
\end{aligned}
$$


$\left.\left.\left\{\operatorname{Min}\left(t_{i j}\right) \mid \mathrm{j} \in H_{2}\right\},\left\{\operatorname{Max}\left(u_{i j}\right) \mid \mathrm{j} \in H_{1}\right\} ; \operatorname{Min}\left(u_{i j}\right) \mid \mathrm{j} \in H_{2}\right\}\right], \quad\left[\left\{\operatorname{Max}\left(v_{i j}\right) \mid \mathrm{j} \in H_{1} ;\left\{\operatorname{Min}\left(v_{i j}\right) \mid \mathrm{j} \in H_{2}\right\}\right.\right.$, $\left.\left.\left\{\operatorname{Max}\left(w_{\mathrm{ij}}\right) \mid \mathrm{j} \in H\right\} ; \operatorname{Min}\left(w_{\mathrm{ij}}\right) \mid \mathrm{j} \in H_{2}\right\}\right]>, \mathrm{j}=1,2, \ldots, n$,

where $H_{1}$ and $H_{2}$ are benefit and cost type attributes, respectively.

Step 3. In the step, we propose three similarity measures between an alternative $Y_{i}, i=1,2, \ldots$, $m$ and the ideal solution $Y^{+}$as given below.

$$
\begin{gathered}
S_{1}\left(Y_{\mathrm{i}}, Y^{+}\right)=1-\frac{1}{12} \sum_{j=1}^{n} w_{\mathrm{j}}\left\langle\begin{array}{l}
\left.\left|a_{i j}-a_{j}^{+}\right|+\left|b_{i j}-b_{j}^{+}\right|+\left|c_{i j}-c_{j}^{+}\right|+\left|d_{i j}-d_{j}^{+}\right|+\left|e_{i j}-e_{j}^{+}\right|+\left|f_{i j}-f_{j}^{+}\right|+\right\rangle \\
\left|r_{i j}-r_{j}^{-}\right|+\left|s_{i j}-s_{j}^{-}\right|+\left|t_{i j}-t_{j}^{-}\right|+\left|u_{i j}-u_{j}^{-}\right|+\left|v_{i j}-v_{j}^{-}\right|+\left|w_{i j}-w_{j}^{-}\right|
\end{array}\right\rangle \\
S M_{2}\left(Y_{\mathrm{i}}, Y^{+}\right)=1-\left\{\begin{array}{l}
\frac{1}{12} \sum_{j=1}^{n} w_{\mathrm{j}}\left[\left(a_{i j}-a_{j}^{+}\right)^{2}+\left(b_{i j}-b_{j}^{+}\right)^{2}+\left(c_{i j}-c_{j}^{+}\right)^{2}+\left(d_{i j}-d_{j}^{+}\right)^{2}+\left(e_{i j}-e_{j}^{+}\right)^{2}+\left(f_{i j}-f_{j}^{+}\right)^{2}+\right)^{1 / 2} \\
\left.\left(r_{i j}-r_{j}^{-}\right)^{2}+\left(s_{i j}-s_{j}^{-}\right)^{2}+\left(t_{i j}-t_{j}^{-}\right)^{2}+\left(u_{i j}-u_{j}^{-}\right)^{2}+\left(v_{i j}-v_{j}^{-}\right)^{2}+\left(w_{i j}-w_{j}^{-}\right)^{2}\right]
\end{array}\right\} \\
Q\left(Y_{\mathrm{i}}, Y^{+}\right)=\sum_{i=1}^{n} w_{i}\left\langle\begin{array}{l}
\sigma^{+} \frac{\min \left(e_{i j}, e_{j}^{+}\right)}{\max \left(a_{i j}, a_{j}^{+}\right)}+\varepsilon^{+} \frac{\min \left(b_{i j}, b_{j}^{+}\right)}{\max \left(b_{i j}, b_{j}^{+}\right)}+\eta^{+} \frac{\min \left(c_{i j}, c_{j}^{+}\right)}{\max \left(c_{i j}, c_{j}^{+}\right)}+\kappa^{+} \frac{\min \left(d_{i j}, d_{j}^{+}\right)}{\max \left(f_{i j}, f_{j}^{+}\right)}+\chi^{-} \frac{\min \left(r_{i j}, r_{j}^{+}\right)}{\max \left(r_{i j}, r_{j}^{+}\right)}+\varepsilon^{-} \frac{\min \left(s_{i j}, s_{j}^{+}\right)}{\max \left(s_{i j}, s_{j}^{+}\right)}+ \\
\eta^{-} \frac{\min \left(t_{i j}, t_{j}^{+}\right)}{\max \left(t_{i j}, t_{j}^{+}\right)}+\kappa^{-} \frac{\min \left(u_{i j}, u_{j}^{+}\right)}{\max \left(u_{i j}, u_{j}^{+}\right)}+\sigma^{-} \frac{\min \left(v_{i j}, v_{j}^{+}\right)}{\max \left(v_{i j}, v_{j}^{+}\right)}+\tau^{-} \frac{\min \left(w_{i j}, w_{j}^{+}\right)}{\max \left(w_{i j}, w_{j}^{+}\right)}
\end{array}\right\rangle
\end{gathered}
$$

Step 4. Using the weighted similarity measures $S M_{1}\left(Y_{\mathrm{i}}, Y^{+}\right), S M_{2}\left(Y_{\mathrm{i}}, Y^{+}\right)$and $Q\left(Y_{\mathrm{i}}, Y^{+}\right)$the ranking order of the alternatives is obtained and the best alternative is selected. The bigger value of $S M_{1}\left(Y_{i}, Y^{+}\right)$reflects the better alternative. Similar result holds for $S M_{2}\left(Y_{i}, Y^{+}\right)$and $Q\left(Y_{i}, Y^{+}\right)$.

\section{Algorithmic representation of the proposed MADM strategies in interval bipolar neutrosophic environment}

We present three MADM strategies in algorithmic form using the following steps:

Step 1. The decision maker provides the interval bipolar neutrosophic decision matrix $\left[\widetilde{D}_{i j}\right]_{m \times n}$.

Step 2. IBN-PIS is computed from the specified interval bipolar neutrosophic decision $\operatorname{matrix}\left[\widetilde{D}_{i j}\right]_{m \times n}$.

Step 3. The weighted similarity measures $S M_{1}\left(Y_{i}, Y^{+}\right), S M_{2}\left(Y_{i}, Y^{+}\right)$and $Q\left(Y_{i}, Y^{+}\right)$between each alternative $Y_{i}, i=1,2, \ldots, m$ and the ideal solution $Y^{+}$are computed respectively, using Eqs. (12), (13) and (14).

Step 4. Rank the alternatives based on the descending order of $S M_{1}\left(Y_{i}, Y^{+}\right), S M_{2}\left(Y_{i}, Y^{+}\right)$and $Q\left(Y_{i}\right.$, $\left.Y^{+}\right), \mathrm{i}=1,2, \ldots, m$.

i. Based on $S M_{1}\left(Y_{i}, Y^{+}\right)$, the highest value of $S M_{1}\left(Y_{i}, Y^{+}\right)$indicates that $Y_{i}, \mathrm{i}=1,2, \ldots, m$ is the best alternative.

ii. Based on $S M_{2}\left(Y_{i}, Y^{+}\right)$, the highest value of $S M_{2}\left(Y_{i}, Y^{+}\right)$reflects that $Y_{i}, \mathrm{i}=1,2, \ldots, m$ is the best alternative.

iii. Based on $Q\left(Y_{i}, Y^{+}\right)$, the highest value of $Q\left(Y_{i}, Y^{+}\right)$signifies that $Y_{i}, \mathrm{i}=1,2, \ldots, m$ is the best alternative.

Step 5. Choose the most desirable alternative. 
Step 6. Stop.

Representation of the proposed strategy is shown in Figure 1.
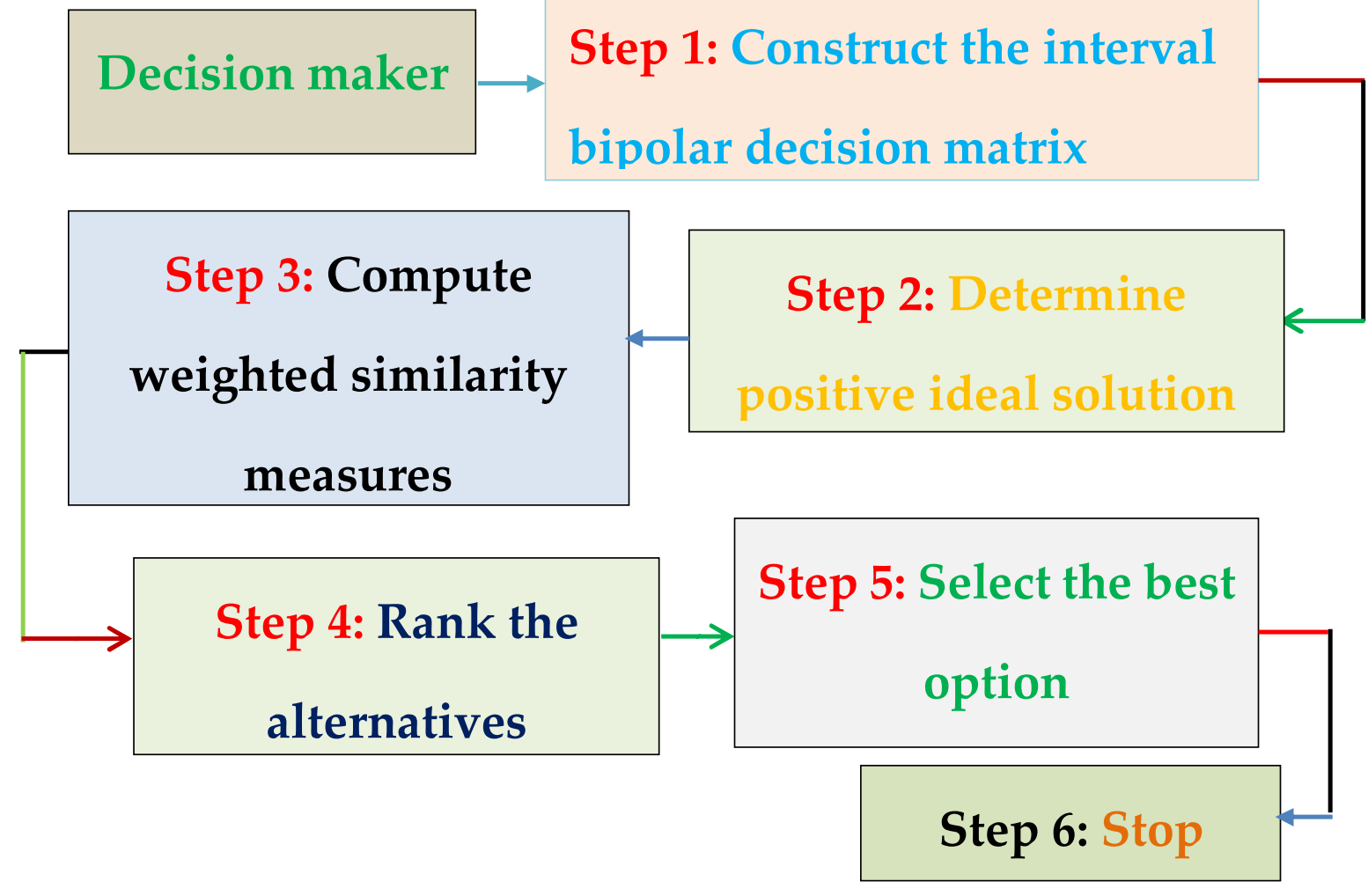

Figure 1. Conceptual representation of the proposed strategies

\section{Illustrative numerical example}

Consider a decision making problem discussed in $[17,19]$ where there are four possible alternatives to invest money namely: a food company $\left(Y_{1}\right)$, a car company $\left(Y_{2}\right)$, a arm company $\left(Y_{3}\right)$, and a computer company $\left(Y_{4}\right)$. The investment company must take a decision based on the three predefined attributes namely: growth analysis $\left(Z_{1}\right)$, risk analysis $\left(Z_{2}\right)$, and environment analysis $\left(Z_{3}\right)$ where $Z_{1}, Z_{2}$ are benefit type and $Z_{3}$ is cost type attributes [21]. Suppose the weight vector of $Z_{1}, Z_{2}$, and $Z_{3}$ is given by $w=\left(w_{1}, w_{2}, w_{3}\right)=(0.35,0.25,0.4)$ [17]. Now the decision making steps are shown as given below.

Step 1. The decision maker evaluates the alternatives $Y_{i}, i=1,2,3,4$ based on the three attributes $Z_{j}, j=1,2,3$ in terms of interval bipolar neutrosophic decision matrix as follows:

$$
\left.\begin{array}{cc}
Z_{1} \\
\left(\begin{array}{cc}
Y_{1} & {[[0.4,0.5],[0.2,0.3],[0.3,0.4],[-0.3,-0.2],[-0.4,-0.3],[-0.5,-0.4]]} \\
Y_{2} & {[[0.6,0.7],[0.1,0.2],[0.2,0.3],[-0.2,-0.1],[-0.3,-0.2],[-0.7,-0.6]]} \\
Y_{3} & {[[0.3,0.6],[0.2,0.3],[0.3,0.4],[-0.3,-0.2],[-0.4,-0.3],[-0.6,-0.3]]} \\
Y_{4} & {[[0.7,0.8],[0.0,0.1],[0.1,0.2],[-0.1,-0.0],[-0.2,-0.1],[-0.8,-0.7]]}
\end{array}\right) \\
& Z_{2} \\
Y_{1} & {[[0.4,0.6],[0.1,0.3],[0.2,0.4],[-0.3,-0.1],[-0.4,-0.2],[-0.6,-0.4]]} \\
Y_{2} & {[[0.6,0.7],[0.1,0.2],[0.2,0.3],[-0.2,-0.1],[-0.3,-0.2],[-0.7,-0.6]]} \\
Y_{3} & {[[0.5,0.6],[0.2,0.3],[0.3,0.4],[-0.3,-0.2],[-0.4,-0.3],[-0.6,-0.5]]} \\
Y_{4} & {[[0.6,0.7],[0.1,0.2],[0.1,0.3],[-0.2-0.1],[-0.3,-0.1],[-0.7,-0.6]]}
\end{array}\right)
$$


15 of 18

$$
\begin{array}{cc}
Z_{3} \\
\left(\begin{array}{cc}
Y_{1} & {[[0.7,0.9],[0.2,0.3],[0.4,0.5],[-0.3,-0.2],[-0.5,-0.4],[-0.9,-0.7]]} \\
Y_{2} & {[[0.3,0.6],[0.3,0.5],[0.8,0.9],[-0.5,-0.3],[-0.9,-0.8],[-0.6,-0.3]]} \\
Y_{3} & {[[0.4,0.5],[0.2,0.4],[0.7,0.9],[-0.4,-0.2],[-0.9,-0.7],[-0.5,-0.4]]} \\
Y_{4} & {[[0.6,0.7],[0.3,0.4],[0.8,0.9],[-0.4,-0.3],[-0.9,-0.8],[-0.7,-0.6]]}
\end{array}\right)
\end{array}
$$

Step 2. Determine the IBN-PIS $\left(Y^{+}\right)$from the interval bipolar neutrosophic decision matrix as follows:

$$
\left\langle\left[a_{1}^{+}, b_{1}^{+}\right],\left[c_{1}^{+}, d_{1}^{+}\right],\left[e_{1}^{+}, f_{1}^{+}\right],\left[r_{1}^{-}, s_{1}^{-}\right],\left[t_{1}^{-}, u_{1}^{-}\right],\left[v_{1}^{-}, w_{1}^{-}\right]\right\rangle=<[0.7,0.8],[0.0,0.1],[0.1,0.2],[-0.3,-0.2],[-0.2,
$$

$-0.1],[-0.5,-0.3]$

$$
\left\langle\left[a_{2}^{+}, b_{2}^{+}\right],\left[c_{2}^{+}, d_{2}^{+}\right],\left[e_{2}^{+}, f_{2}^{+}\right],\left[r_{2}^{-}, s_{2}^{-}\right],\left[t_{2}^{-}, u_{2}^{-}\right],\left[v_{2}^{-}, w_{2}^{-}\right]\right\rangle=<[0.6,0.7],[0.1,0.2],[0.1,0.3],[-0.3,-0.2],[-0.3,
$$

$-0.1],[-0.6,-0.4]$

$$
\left\langle\left[a_{3}^{+}, b_{3}^{+}\right],\left[c_{3}^{+}, d_{3}^{+}\right],\left[e_{3}^{+}, f_{3}^{+}\right],\left[r_{3}^{-}, s_{3}^{-}\right],\left[t_{3}^{-}, u_{3}^{-}\right],\left[v_{3}^{-}, w_{3}^{-}\right]\right\rangle=<[0.3,0.5],[0.3,0.5],[0.8,0.9],[-0.3,-0.2],[-0.9,
$$

$-0.8],[-0.9,-0.7]$.

Step 3. We calculate the similarity measures $S M_{1}\left(Y_{i}, Y^{+}\right), S M_{2}\left(Y_{i}, Y^{+}\right)$and $Q\left(Y_{i}, Y^{+}\right), i=1,2,3,4$ between each alternative $Y_{i}, i=1,2,3,4$ and ideal solution $Y^{+}$as follows:

$$
\begin{aligned}
& S M_{1}\left(Y_{1}, Y^{+}\right)=0.8354, S M_{1}\left(Y_{2}, Y^{+}\right)=0.9050, S M_{1}\left(Y_{3}, Y^{+}\right)=0.8812, S M_{1}\left(Y_{4}, Y^{+}\right)=0.9208, \\
& S M_{2}\left(Y_{1}, Y^{+}\right)=0.7798, S M_{2}\left(Y_{2}, Y^{+}\right)=0.8646, S M_{2}\left(Y_{3}, Y^{+}\right)=0.8599, S M_{2}\left(Y_{4}, Y^{+}\right)=0.8655, \\
& Q\left(Y_{1}, Y^{+}\right)=0.6728, Q\left(Y_{2}, Y^{+}\right)=0.7443, Q\left(Y_{3}, Y^{+}\right)=0.7165, Q\left(Y_{4}, Y^{+}\right)=0.8229 .
\end{aligned}
$$

Step 4. We rank the alternatives based on the descending order of $S M_{1}\left(Y_{i}, Y^{+}\right), S M_{2}\left(Y_{i}, Y^{+}\right)$and $Q\left(Y_{i}\right.$, $\left.Y^{+}\right), \mathrm{i}=1,2,3$, 4 as follows:

(i) We observe that $S M_{1}\left(Y_{4}, Y^{+}\right)>S M_{1}\left(Y_{2}, Y^{+}\right)>S M_{1}\left(Y_{3}, Y^{+}\right)>S M_{1}\left(Y_{1}, Y^{+}\right)$, the ranking order of the alternatives is obtained as $Y_{4}>Y_{2}>Y_{3}>Y_{1}$. Therefore, $Y_{4}$ is the best option.

(ii) We see that $S M_{2}\left(Y_{4}, Y^{+}\right)>S M_{2}\left(Y_{2}, Y^{+}\right)>S M_{2}\left(Y_{3}, Y^{+}\right)>S M_{2}\left(Y_{1}, Y^{+}\right)$, the ranking order of the alternatives is obtained as $Y_{4}>Y_{2}>Y_{3}>Y_{1}$. Consequently, $Y_{4}$ is the best choice.

(iii) We see that $Q\left(Y_{4}, Y^{+}\right)>Q\left(Y_{2}, Y^{+}\right)>Q\left(Y_{3}, Y^{+}\right)>Q\left(Y_{1}, Y^{+}\right)$. Thus the ranking order of the companies is $Y_{4}>Y_{2}>Y_{3}>Y_{1}$. Obviously, $Y_{4}$ is the most desirable alternative.

Step 5. Thereby, the computer company $\left(Y_{4}\right)$ is the most suitable option to invest money.

Note 1. We observe that similarity measures $S M_{1}\left(Y_{i}, Y^{+}\right), S M_{2}\left(Y_{i}, Y^{+}\right)$and $Q\left(Y_{i}, Y^{+}\right), i=1,2,3$, 4 yield same ranking order. Based on the proposed similarity measures we find that the computer company $\left(Y_{4}\right)$ is the most suitable company to spend money. The comparison of the proposed similarity measures $S M_{1}\left(Y_{i}, Y^{+}\right), S M_{2}\left(Y_{i}, Y^{+}\right)$and $Q\left(Y_{i}, Y^{+}\right), i=1,2,3,4$ is presented in the Fig. 2. 


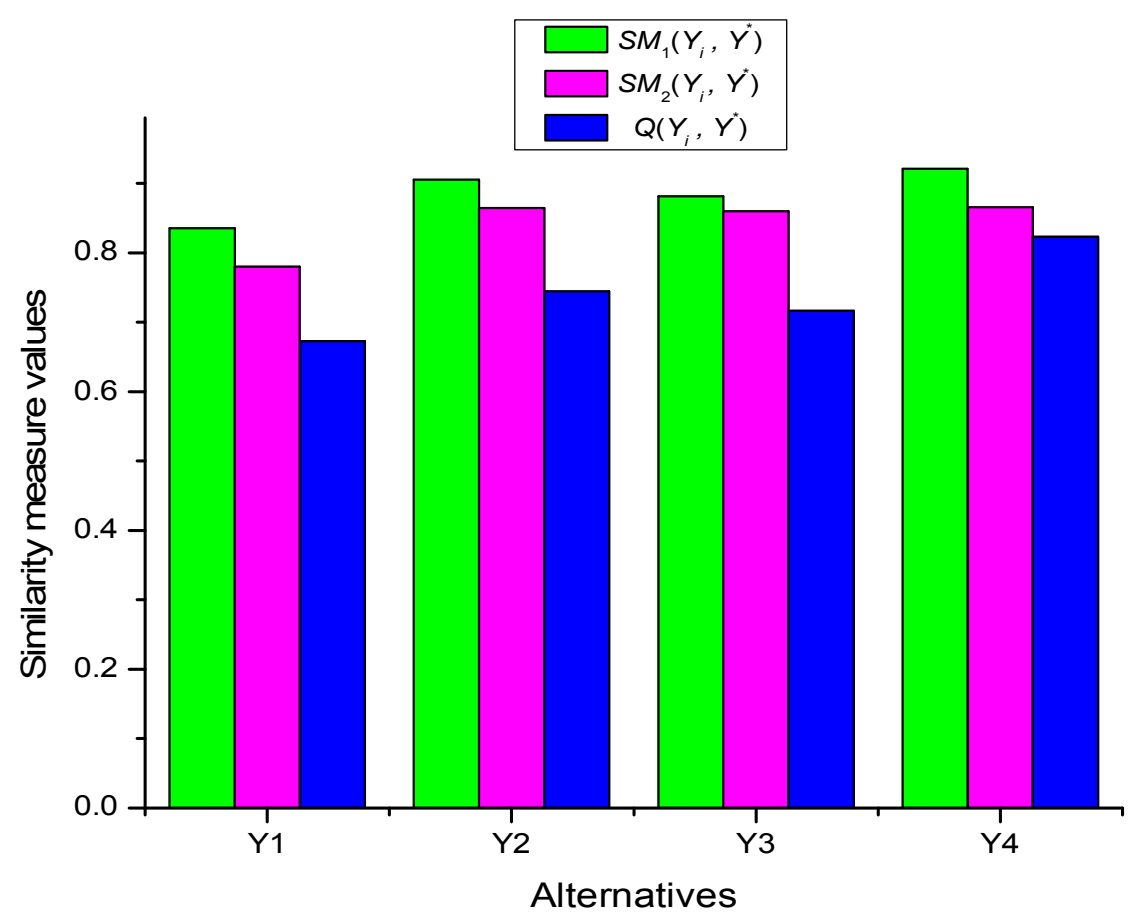

Figure 2. Comparison of the results obtained from proposed strategies

Note 2. Mahmood et al. [17] obtained the best alternative via score function in interval bipolar neutrosophic environment on the basis of weighted arithmetic average operator. Pramanik et al. [19] studied weight cross entropy measure to find the best option. It is to be observed that proposed strategies obtained the same ranking order as the method discussed by Mahmood et al. [17]. However, Pramanik et al. [19] obtained different ranking order. We present the comparison of the results obtained from the different strategies in Table 1.

Table 1. Comparison of the proposed strategy with other existing strategies

\begin{tabular}{|c|c|c|c|}
\hline Measure strategies & Measure values & Ranking order & Best option \\
\hline Proposed $S M_{1}\left(Y_{i}, Y^{+}\right)$ & $\begin{array}{l}S M_{1}\left(Y_{i}, Y^{+}\right)=0.8354 \\
S M_{1}\left(Y_{2}, Y^{+}\right)=0.9050 \\
S M_{1}\left(Y_{3}, Y^{+}\right)=0.8812 \\
S M_{1}\left(Y_{4}, Y^{+}\right)=0.9208\end{array}$ & $Y_{4}>Y_{2}>Y_{3}>Y_{1}$ & $Y_{4}$ \\
\hline Proposed $S M_{2}\left(Y_{\mathrm{i}}, \mathrm{Y}^{+}\right)$ & $\begin{array}{l}S M_{2}\left(Y_{i}, Y^{+}\right)=0.7798 \\
S M_{2}\left(Y_{2}, Y^{+}\right)=0.8646 \\
S M_{2}\left(Y_{3}, Y^{+}\right)=0.8599 \\
S M_{2}\left(Y_{4}, Y^{+}\right)=0.8655\end{array}$ & $Y_{4}>Y_{2}>Y_{3}>Y_{1}$ & $Y_{4}$ \\
\hline Proposed $Q\left(Y_{i}, Y^{+}\right)$ & $\begin{array}{l}Q\left(Y_{i}, Y^{+}\right)=0.6728 \\
Q\left(Y_{2}, Y^{+}\right)=0.7443 \\
Q\left(Y_{3}, Y^{+}\right)=0.7165 \\
Q\left(Y_{4}, Y^{+}\right)=0.8229\end{array}$ & $Y_{4}>Y_{2}>Y_{3}>Y_{1}$ & $Y_{4}$ \\
\hline Weighted cross entropy & $C_{I B}\left(Y_{\mathrm{i},}, \mathrm{Y}^{+}\right)_{w}=0.0606$ & $Y_{1}>Y_{3}>Y_{4}>Y_{2}$ & $Y_{2}$ \\
\hline
\end{tabular}


measure [19]

Mahmood et al.'s

method [17]

$$
\begin{aligned}
& C_{I B}\left(Y_{2}, \mathrm{Y}^{+}\right)_{w}=0.0286 \\
& C_{I B}\left(Y_{3}, \mathrm{Y}^{+}\right)_{w}=0.0426 \\
& C_{I B}\left(Y_{4}, \mathrm{Y}^{+}\right)_{w}=0.0423
\end{aligned}
$$

$S\left(\beta_{1}\right)=0.2574$

$S\left(\beta_{2}\right)=-0.837$

$S\left(\beta_{3}\right)=-0.454$

$S\left(\beta_{4}\right)=0.8050$

\section{Conclusion}

We have defined Hamming and Euclidean distance measures of IBNSs and proved their basic properties. Using the proposed distance measures, we have developed new similarity measures for MADM problems. We have also defined similarity measures using the maximum and minimum operators of IBNSs and proved their basic properties. Then, we have developed three novel strategies to solve MADM problems in interval bipolar neutrosophic setting based on the proposed similarity measures. Comparison analysis with other existing strategies is presented. We hope that the proposed similarity measures of IBNSs can be applied in the field of practical decision making such as brick selection [22], fault diagnosis [23], logistics center location selection [24], renewable energy project selection [25], teacher selection [26], weaver selection [27], etc.

Author Contributions: "S. Pramanik and P.P. Dey conceived and designed the experiments; S. Pramanik performed the experiments; F. Smarandache analyzed the data; S. Pramanik and P.P. Dey wrote the paper."

Conflicts of Interest: "The authors declare no conflict of interest."

\section{References}

1. Zhang, W.R. Bipolar fuzzy sets. In Proceedings of the IEEE World Congress on Computational Science (FuzzIEEE), Anchorage, Alaska, 1998, 835-840, DOI:10.1109/FUZZY.1998.687599.

2. Zhang, W.R. Bipolar fuzzy sets and relations: a computational framework for cognitive modeling and multiagent decision analysis. In Proceedings of IEEE Conference, 1994, 305-309, DOI: 10.1109/IJCF.1994.375115.

3. Ezhilmaran D., Shankar, K. Morphism of bipolar intuitionistic fuzzy graphs. J. Discrete Math. Sciences Crypt. 2015, 18(5), 605-621.

4. Deli,I.; Ali, M., Smarandache, F. Bipolar neutrosophic sets and their application based on multi-criteria decision making problems. In International conference on advanced mechatronic systems (ICAMechS) (IEEE, August, 2015), 2015, 249-254.

5. Zadeh, L.A. Fuzzy sets. Inf. Control. 1965, 8(3), 338-353.

6. Atanassov, K.T. Intuitionistic fuzzy sets. Fuzzy Sets \& Systs. 1986, 20, 87-96.

7. Smarandache, F. A unifying field of logics. Neutrosophy: Neutrosophic probability, set and logic; American Research Press: Rehoboth, 1998.

8. Dey, P.P.; Pramanik, S.; Giri, B.C. TOPSIS for solving multi-attribute decision making problems under bi-polar neutrosophic environment. In New Trends in Neutrosophic Theory and Applications; Smarandache, F.; Pramanik, S.; Eds., Pons asbl: Brussells, 2016, 65-77, ISBN 978-1-59973-498-9.

9. Pramanik, S.; Dey, P.P.; Giri, B.C.; Smarandache, F. Bipolar neutrosophic projection based models for solving multi-attribute decision making problems. Neut. Sets $\mathcal{E}$ Systs. 2017, 15, 74-83, DOI:10.5281/zenedo.570936.

10. Ye, J. Another form of correlation coefficient between single valued neutrosophic sets and its multiple attribute decision making method. Neut. Sets \& Systs. 2013, 1, 8-11.

11. Ye, J.; Zhang Q. Single valued neutrosophic similarity measures for multiple attribute decision making. Neut. Sets E Systs. 2014, 2, 48-54.

12. Ye, J. Similarity measure of refined single-valued neutrosophic sets and its multicriteria decision making method. Neut. Sets $\mathcal{E}$ Systs. 2016, 12, 41-44. 
13. Deli, I., Subas, Y. A. Multiple criteria decision making methodon single valued bipolar neutrosophic set based on correlation coefficient similarity measure, In International Conference on mathematics and mathematics education (ICMME-2016), Frat University, Elazg, Turkey May 12-14, 2016.

14. . Şahin, I., Deli, M.; V. Uluçay. Jaccard vector similarity measure of bipolar neutrosophic set based on multi-criteria decision making, in International Conference on Natural Science and Engineering (ICNASE'16), Killis, March 19-20, 2016.

15. V. Uluçay, I. Deli, M. Şahin, Similarity measures of bipolar neutrosophic sets and their application to multiple criteria decision makin., Neural Comput. E Applic. 2016, DOI 10.1007/s00521-016-2479-1.

16. Deli, I.; Y.A. Subas; F. Smarandache, F. M. Ali, Interval valued bipolar neutrosophic sets and their application in pattern recognition. https://www.researchgate.net/publication/289587637. Retrieved on October 9, 2017.

17. Mahmood, T.; Ye, J. and Q. Khan, Bipolar interval neutrosophic set and its application in multicriteria decision making, https://archive.org/details/BipolarIntervalNeutrosophicSet. Retrieved on October 9, 2017.

18. Wang, H.; F. Smarandache, Y.Q. Zhang and R. Sunderraman, Interval neutrosophic sets and logic: theory and applications in computing, Hexis, Arizona, U.S.A, 2005.

19. Pramanik, S.; Dey, P.P.; Smarandache, F.; Ye, J. Cross entropy measures of bipolar and interval bipolar neutrosophic sets and their application for multi-attribute decision making, Axioms 2018, 7(2), 1-24, DOI: 10.3390/axioms7020021.

20. Pramanik, S.; Dey, P.P.; Smarandache, F. Correlation coefficient measures of interval bipolar neutrosophic sets for solving multi-attribute decision making problems, Neut. Sets $\mathcal{E}$ Systs. 19. 2018 (In press).

21. J. Ye, Similarity measures between interval neutrosophic sets and their applications in multicriteria decision-making, J. Intell. Fuzzy Syst. 2014, 26, 165-172.

22. Mondal, K.; Pramanik, S. Intuitionistic fuzzy multi criteria group decision making approach to quality-brick selection problem. J. Appl. Quant. Methods 2014, 9, 35-50.

23. Ye, J. Fault diagnoses of steam turbine using the exponential similarity measure of neutrosophic numbers. J. Intell. Fuzzy Syst. 2016, 30, 1927-1934.

24. Pramanik, S.; Dalapati, S.; Roy, T.K. Logistics center location selection approach based on neutrosophic multi-criteria decision making. In New Trends in Neutrosophic Theory and Applications; Smarandache, F., Pramanik, S., Eds.; Pons Asbl: Brussels, Belgium, 2016; Volume 1, pp. 161-174, ISBN 978-1-59973-498-9.

25. San Cristóbal, J. R. Multi-criteria decision-making in the selection of a renewable energy project in Spain: The VIKOR method. Ren. Energy, 2011, 36, 498-502.

26. Pramanik, S.; Mukhopadhyaya, D. Grey relational analysis-based intuitionistic fuzzy multi-criteria group decision-making approach for teacher selection in higher education. Int. J. Comput. Appl. 2011, 34, 21-29, doi:10.5120/4138-5985.

27. Dey, P.P.; Pramanik, S.; Giri, B.C. Multi-criteria group decision making in intuitionistic fuzzy environment based on grey relational analysis for weaver selection in Khadi institution. J. Appl. Quant. Methods 2015, 10, 1-14. 